\title{
Radiative corrections to polarization observables in elastic electron-deuteron scattering in leptonic variables
}

\author{
G. I. Gakh, M. I. Konchatnij, and N. P. Merenkov* \\ Kharkov Institute of Physics and Technology \\ 61108, Akademicheskaya, 1, Kharkov, Ukraine
}

\begin{abstract}
The model-independent QED radiative corrections to polarization observables in elastic scattering of unpolarized and longitudinally-polarized electron beam by the deuteron target have been calculated in leptonic variables. The experimental setup when the deuteron target is arbitrarily polarized is considered and the procedure for applying derived results to the vector or tensor polarization of the recoil deuteron is discussed. The basis of the calculations consists of the account for all essential Feynman diagrams which results in the form of the Drell-Yan representation for the cross-section and use of the covariant parametrization of the deuteron polarization state. The numerical estimates of the radiative corrections are given for the case when event selection allows the undetected particles (photons and electron-positron pairs) and the restriction on the lost invariant mass is used.
\end{abstract}

\section{INTRODUCTION}

The process of the elastic electron-deuteron scattering has been a long time a reaction which is used for the investigation of the electromagnetic structure of the deuteron. These investigations, both theoretical and experimental, can help to clarify a number of the important problems: the properties of the nucleon-nucleon interaction, non-nucleonic degrees of freedom in nuclei (such as the meson exchange currents, the isobar configurations), as well as the importance of the relativistic effects (see, for example, the recent reviews on the deuteron $[1-4])$.

The electromagnetic structure of the deuteron as a bound two-nucleon system with spinone is completely determined by three functions of one variable, the four-momentum transfer

\footnotetext{
* Electronic address: merenkov@kipt.kharkov.ua
} 
squared $Q^{2}$. These are the so-called electromagnetic form factors of the deuteron: $G_{C}$ (the charge monopole), $G_{M}$ (the magnetic dipole) and $G_{Q}$ (the charge quadrupole). They are real functions in the space-like region of the four-momentum transfer squared (the scattering channel as, for example, the elastic electron-deuteron scattering) and complex functions in the time-like region (the annihilation channel as, for example, $e^{+}+e^{-} \rightarrow D+\bar{D}$ ). So, the main experimental problem is to determine the electromagnetic deuteron form factors with a high accuracy and in a wide range of the variable $Q^{2}$. A recent review of past and future measurements of the elastic electromagnetic deuteron form factors is given in Ref. [5].

Note also that the deuteron is used as an effective neutron target in studies of the neutron electromagnetic form factors [6] and the spin structure functions of the neutron in the deepinelastic scattering [7].

The knowledge of the deuteron electromagnetic properties means that we can calculate its form factors from the first principles. To do it, in the non relativistic approach, we need the deuteron wave function and form factors of the nucleon. The last ones are known from the analysis of the experimental data on the elastic $e N$-scattering. It was found that for the agreement with experiment at small momentum transfers it is sufficient to take into account one-body current. The meson exchange currents and the isobar configurations become significant at large momentum transfers. The manifestation of the quarks inside the deuteron was not found at present. Note that each deuteron form factor may be sensitive to some specific contribution. Thus, for example, the deuteron charge form factor $G_{C}$ is particularly interesting for the understanding of the role of the meson exchange currents. So, it is necessary to separate the three deuteron form factors. Measurements of the unpolarized cross section yield the structure functions $A\left(Q^{2}\right)$ and $B\left(Q^{2}\right)$ : they can be separately determined by variation of the scattered electron angle $\theta_{e}$ for a given momentum transfer squared $Q^{2}$ to the deuteron. So, all three form factors can be separated, when either the tensor analyzing power $T_{20}$ or the recoil deuteron polarization $t_{20}$ is also measured (in both cases the electron beam is unpolarized). This has prompted development of both polarized deuterium targets for use with internal or external beams and polarimeters for measuring the polarization of recoil hadrons [8]. Both types of experiment result in the same combinations of form factors.

The measurement of the polarization observables in elastic ed-scattering can be done at present with the help of the internal or external targets.

1. The internal targets were used at storage rings in form of the polarized deuteron gas 
targets [9-13]. In order to get the required luminosity it is necessary to have high-intensity electron beam since the density of such targets is very small.

2. In the experiments on elastic ed-scattering with external targets, the measurement of the polarization of the scattered deuteron is used [14-16]. In this case the high-intensity electron beam is also necessary since the polarization measurement requires polarimeter. This procedure leads to the second scattering which decreases the event number very essentially.

Current experiments at modern accelerators reached a new level of precision and this circumstance requires a new approach to data analysis and inclusion of all possible systematic uncertainties. One of the important source of such uncertainties is the electromagnetic radiative effects caused by physical processes which take place in higher orders of the perturbation theory with respect to the electromagnetic interaction.

While the radiative corrections have been taken into account for the unpolarized cross section, the radiative corrections for the polarization observables in the elastic electrondeuteron scattering at large momentum transfer are not known at present [17]. Thus, for example, in the experiment on precise measurement of the deuteron elastic structure function $A\left(Q^{2}\right)$ (at $Q=0.66-1.8 \mathrm{GeV}$ ), the radiative corrections (about $20 \%$ ) due to loses in the radiative tail were calculated according to the paper [18]. On the other side, the authors of recent experiments $[12,13,16]$ on measuring the polarization observables did not present the evidence about taking into account the radiative corrections.

The importance of the taking into account the radiative corrections can be seen on the example of the discrepancy between the Rosenbluth [19] and the polarization transfer methods [20] for determination of the ratio of the electric to magnetic proton form factors. For a given value of $Q^{2}$, it is sufficient to measure the unpolarized elastic electron-nucleon scattering cross section for two values of $\varepsilon$ (virtual photon polarization parameter) to determine the $G_{M}$ and $G_{E}$ form factors (the Rosenbluth method). But the measurement of the polarization observables in this reaction (using the longitudinally polarized electron beam) allows to determine the ratio $G_{E}$ to $G_{M}$ [20]. Two experimental set ups were used, namely: measurement of the asymmetry on the polarized target or measurement of the recoil-proton polarization (the polarization transfer method).

Recent experiments show that the extracted ratio $G_{E p} / G_{M p}$, using the Rosenbluth and polarization transfer methods, are incompatible at large $Q^{2}[21,22]$. This discrepancy is a serious problem as it generates confusion and doubt about the whole methodology of 
lepton scattering experiments [23]. One plausible explanation of this problem is two-photon exchange effects [24]. The data are consistent with simple estimates of the two-photon contributions to explain the discrepancy (see, e.g., [25] and references therein).

The precise calculation of the radiative corrections is also important for the study of the two-photon exchange effects in the elastic electron-deuteron scattering. Earlier it was observed [26-29] that the relative role of the two-photon exchange can increase significantly in the region of large $Q^{2}$ due to the steep decrease of the deuteron form factors as functions of the $Q^{2}$ variable. Since one- and two-photon amplitudes have very different spin structures, the polarization phenomena have to be more sensitive to the interference effects than the differential cross section (with unpolarized particles).

An attempt to evaluate the presence of two intermediate hard photon in box diagrams using the existing data on the elastic electron-deuteron scattering was done in Ref.[30]. The authors searched a deviation from the linear dependence in $\cot ^{2}\left(\theta_{e} / 2\right)$ of the cross section, using a Rosenbluth fit, which has been parameterized in a model independent way according to crossing symmetry considerations.

The recent calculations of the two-photon contribution to the structure functions and polarization observables in the elastic scattering of longitudinally polarized electron on polarized deuteron have been done in Ref. [31] (the references on earlier papers can be found here).

The radiative corrections to deep-inelastic scattering of unpolarized and longitudinally polarized electron beam on polarized deuteron target was considered in Ref. [32] for the particular case of the deuteron polarization (which can be obtained from the general covariant spin-density matrix [33] when spin functions are the eigenvectors of the spin projection operator). The leading-log model-independent radiative corrections in deep-inelastic scattering of unpolarized electron beam off the tensor polarized deuteron target have been considered in Ref. [34]. The calculation was based on the covariant parametrization of the deuteron quadrupole polarization tensor and use of the Drell-Yan like representation in electrodynamics. The model-independent QED radiative corrections to the polarization observables in the elastic scattering of the unpolarized and longitudinally polarized electron beam by the polarized deuteron target in the hadronic variables have been done in Ref. [35].

In present paper we calculate the model-independent QED radiative corrections in leptonic variables to the polarization observables in the elastic scattering of unpolarized and 
longitudinally-polarized electron beam by deuteron target

$$
e^{-}\left(k_{1}\right)+D\left(p_{1}\right) \rightarrow e^{-}\left(k_{2}\right)+D\left(p_{2}\right),
$$

where the four-momenta of the corresponding particles are indicated in the brackets. The experimental setup when the deuteron target is arbitrarily polarized is considered and the procedure for applying derived results to the vector or tensor polarization of the recoil deuteron is discussed. The basis of the calculations consists of the account for all essential Feynman diagrams which results in the form of the Drell-Yan representation for the cross section and use of the covariant parametrization of the deuteron polarization state. The numerical estimates of the radiative corrections are given for the case when event selection allows the undetected particles (photons and electron-positron pairs) and the restriction on the lost invariant mass is used.

\section{BORN APPROXIMATION}

From the theoretical point of view, different polarization observables in the process of the elastic electron-deuteron scattering have been investigated in many papers (see, for example, Refs. [36-41]. The polarization observables were expressed in terms of the deuteron electromagnetic form factors. An up-to-date status of the experimental and theoretical research into the deuteron structure can be found in reviews [2, 4]. Here, we reproduce most of these results using the method of covariant parametrization of the deuteron polarization state in terms of the particle four-momenta and demonstrate the advantage of such approach.

Consider the process of elastic scattering of polarized electron beam by polarized deuteron target. In the one-photon-exchange approximation, we define the cross section of the process (1), in terms of the contraction of the leptonic $L_{\mu \nu}$ and hadronic $H_{\mu \nu}$ tensors (we neglect the electron mass wherever possible), as follows

$$
d \sigma=\frac{\alpha^{2}}{2 V q^{4}} L_{\mu \nu}^{B} H_{\mu \nu} \frac{d^{3} k_{2}}{\varepsilon_{2}} \frac{d^{3} p_{2}}{E_{2}} \delta\left(k_{1}+p_{1}-k_{2}-p_{2}\right),
$$

where $V=2 k_{1} \cdot p_{1}, \varepsilon_{2}$ and $E_{2}$ are the energies of the scattered electron and recoil deuteron, respectively, and $q=k_{1}-k_{2}=p_{2}-p_{1}$ is the four-momentum of the heavy virtual photon that probes the deuteron structure. In the case of longitudinally polarized electron beam we have for the leptonic tensor, in the Born approximation, following expression

$$
L_{\mu \nu}^{B}=q^{2} g_{\mu \nu}+2\left(k_{1 \mu} k_{2 \nu}+k_{2 \mu} k_{1 \nu}\right)+2 i P_{e}\left(\mu \nu q k_{1}\right)
$$




$$
(\mu \nu a b)=\varepsilon_{\mu \nu \lambda \rho} a_{\lambda} b_{\rho}, \varepsilon_{1230}=1
$$

where $P_{e}$ is the degree of the electron beam polarization (further we assume that the electron beam is completely polarized and consequently $P_{e}=1$ ).

The hadronic tensor can be expressed via the deuteron electromagnetic current $J_{\mu}$, describing the transition $\gamma^{*} D \rightarrow D$, as

$$
H_{\mu \nu}=J_{\mu} J_{\nu}^{*}
$$

Using requirements of the Lorentz invariance, current conservation, parity and timereversal invariances of the hadron electromagnetic interaction, the general form of the electromagnetic current for the spin-one deuteron is completely described by three form factors and it can be written as [20]

$$
\begin{array}{r}
J_{\mu}=\left(p_{1}+p_{2}\right)_{\mu}\left[-G_{1}\left(Q^{2}\right) U_{1} \cdot U_{2}^{*}+\right. \\
\left.\frac{G_{3}\left(Q^{2}\right)}{M^{2}}\left(U_{1} \cdot q U_{2}^{*} \cdot q-\frac{q^{2}}{2} U_{1} \cdot U_{2}^{*}\right)\right]+ \\
G_{2}\left(Q^{2}\right)\left(U_{1 \mu} U_{2}^{*} \cdot q-U_{2 \mu}^{*} U_{1} \cdot q\right),
\end{array}
$$

where $U_{1 \mu}$ and $U_{2 \mu}$ are the polarization four-vectors for the initial and final deuteron states, $M$ is the deuteron mass. The functions $G_{i}\left(Q^{2}\right)(i=1,2,3)$ are the deuteron electromagnetic form factors depending only upon the virtual photon four-momentum squared. Due to the current hermiticity the form factors $G_{i}\left(Q^{2}\right)$ are real functions in the region of the space-like momentum transfer. We use here the convention $Q^{2}=-q^{2}$.

These form factors can be related to the standard deuteron form factors: $G_{C}$ (the charge monopole), $G_{M}$ (the magnetic dipole) and $G_{Q}$ (the charge quadrupole). These relations are

$$
\begin{array}{r}
G_{M}=-G_{2}, G_{Q}=G_{1}+G_{2}+2 G_{3} \\
G_{C}=\frac{2}{3} \eta\left(G_{2}-G_{3}\right)+\left(1+\frac{2}{3} \eta\right) G_{1}, \quad \eta=\frac{Q^{2}}{4 M^{2}} .
\end{array}
$$

The standard form factors have the following normalization:

$$
G_{C}(0)=1, G_{M}(0)=\left(M / m_{n}\right) \mu_{d}, G_{Q}(0)=M^{2} Q_{d},
$$

where $m_{n}$ is the nucleon mass, $\mu_{d}\left(Q_{d}\right)$ is deuteron magnetic (quadrupole) moment and their values are: $\mu_{d}=0.857$ [42], $Q_{d}=0.2859 \mathrm{fm}^{2}$ [43]. 
If we write down the electromagnetic current in the following form $J_{\mu}=J_{\mu \alpha \beta} U_{1 \alpha} U_{2 \beta}^{*}$, then the $H_{\mu \nu}$ tensor can be written as

$$
H_{\mu \nu}=J_{\mu \alpha \beta} J_{\nu \sigma \gamma}^{*} \rho_{\alpha \sigma}^{i} \rho_{\gamma \beta}^{f}
$$

where $\rho_{\alpha \sigma}^{i}\left(\rho_{\gamma \beta}^{f}\right)$ is the spin-density matrix of the initial (final) deuteron.

Since we consider the case of a polarized deuteron target and unpolarized recoil deuteron, the hadronic tensor $H_{\mu \nu}$ can be expanded according to the polarization state of the initial deuteron as follows:

$$
H_{\mu \nu}=H_{\mu \nu}(0)+H_{\mu \nu}(V)+H_{\mu \nu}(T)
$$

where the spin-independent tensor $H_{\mu \nu}(0)$ corresponds to the case of unpolarized initial deuteron and the spin-dependent tensor $H_{\mu \nu}(V)\left(H_{\mu \nu}(T)\right)$ describes the case when the deuteron target has vector (tensor) polarization.

We consider the general case of the initial deuteron polarization state which is described by the spin-density matrix. We use the following general expression for the deuteron spindensity matrix in the coordinate representation [44]

$$
\rho_{\alpha \beta}^{i}=-\frac{1}{3}\left(g_{\alpha \beta}-\frac{p_{1 \alpha} p_{1 \beta}}{M^{2}}\right)+\frac{i}{2 M}\left(\alpha \beta s p_{1}\right)+Q_{\alpha \beta},
$$

where $s_{\mu}$ is the polarization four-vector describing the vector polarization of the deuteron target $\left(p_{1} \cdot s=0, s^{2}=-1\right)$ and $Q_{\mu \nu}$ is the tensor describing the tensor (quadrupole) polarization of the initial deuteron $\left(Q_{\mu \nu}=Q_{\nu \mu}, Q_{\mu \mu}=0, p_{1 \mu} Q_{\mu \nu}=0\right)$. In the laboratory system (initial deuteron rest frame) all time components of the tensor $Q_{\mu \nu}$ are zero and the tensor polarization of the deuteron target is described by five independent space components $\left(Q_{i j}=Q_{j i}, Q_{i i}=0, i, j=x, y, z\right)$. In the Appendix B we give the relation between the elements of the deuteron spin-density matrix in the helicity and spherical tensor representations and the ones in the coordinate representation. We give also the relation between the polarization parameters $s_{i}, Q_{i j}$ and the population numbers $n_{+}, n_{-}$and $n_{0}$ describing the polarized deuteron target which is often used in the spin experiments.

In this paper we assume that the polarization of the recoil deuteron is not measured. So, its spin-density matrix can be written as

$$
\rho_{\alpha \beta}^{f}=-\left(g_{\alpha \beta}-\frac{p_{2 \alpha} p_{2 \beta}}{M^{2}}\right)
$$


The spin-independent tensor $H_{\mu \nu}(0)$ describes unpolarized initial and final deuterons and it has the following general form

$$
\begin{aligned}
& H_{\mu \nu}(0)=-W_{1}\left(Q^{2}\right) \tilde{g}_{\mu \nu}+\frac{W_{2}\left(Q^{2}\right)}{M^{2}} \tilde{p}_{1 \mu} \tilde{p}_{1 \nu}, \\
& \tilde{g}_{\mu \nu}=g_{\mu \nu}-\frac{q_{\mu} q_{\nu}}{q^{2}}, \quad \tilde{p}_{1 \mu}=p_{1 \mu}-\frac{p_{1} \cdot q}{q^{2}} q_{\mu} .
\end{aligned}
$$

Two real structure functions $W_{1,2}\left(Q^{2}\right)$ have the following expressions in terms of the deuteron electromagnetic form factors

$$
\begin{array}{r}
W_{1}\left(Q^{2}\right)=\frac{2}{3} Q^{2}(1+\eta) G_{M}^{2}, \\
W_{2}\left(Q^{2}\right)=4 M^{2}\left(G_{C}^{2}+\frac{2}{3} \eta G_{M}^{2}+\frac{8}{9} \eta^{2} G_{Q}^{2}\right) .
\end{array}
$$

In the considered case the spin-dependent tensor $H_{\mu \nu}(V)$, that describes the vector polarized initial deuteron and unpolarized final deuteron, can be written as

$$
\begin{gathered}
H_{\mu \nu}(V)=\frac{i}{M} S_{1}(\mu \nu s q)+\frac{i}{M^{3}} S_{2}\left[\tilde{p}_{1 \mu}\left(\nu s q p_{1}\right)-\right. \\
\left.-\tilde{p}_{1 \nu}\left(\mu s q p_{1}\right)\right]+\frac{1}{M^{3}} S_{3}\left[\tilde{p}_{1 \mu}\left(\nu s q p_{1}\right)+\tilde{p}_{1 \nu}\left(\mu s q p_{1}\right)\right],
\end{gathered}
$$

where three real structure functions $S_{i}\left(Q^{2}\right), i=1-3$ can be expressed in terms of the deuteron electromagnetic form factors. They are

$$
\begin{gathered}
S_{1}\left(Q^{2}\right)=M^{2}(1+\eta) G_{M}^{2}, S_{3}\left(Q^{2}\right)=0, \\
S_{2}\left(Q^{2}\right)=M^{2}\left[G_{M}^{2}-2\left(G_{C}+\frac{\eta}{3} G_{Q}\right) G_{M}\right] .
\end{gathered}
$$

The third structure function $S_{3}\left(Q^{2}\right)$ is zero since deuteron form factors are real functions in the elastic scattering (space-like momentum transfers). In the time-like region of the momentum transfers (for annihilation processes, for example, $e^{-}+e^{+} \rightarrow D+\bar{D}$ ), where the form factors are complex functions, the structure function $S_{3}\left(Q^{2}\right)$ is not zero and it is determined by the imaginary part of the form factors, namely: $S_{3}\left(Q^{2}\right)=2 M^{2} \operatorname{Im}\left(G_{C}-\right.$ $\left.\eta / 3 G_{Q}\right) G_{M}^{*}$ (in this case $Q^{2}$ is the square of the virtual photon four-momentum).

In the case of tensor-polarized deuteron target the general structure of the spin-dependent tensor $H_{\mu \nu}(T)$ can be written in terms of five structure functions as follows

$$
\begin{array}{r}
H_{\mu \nu}(T)=V_{1}\left(Q^{2}\right) \bar{Q} \tilde{g}_{\mu \nu}+V_{2}\left(Q^{2}\right) \frac{\bar{Q}}{M^{2}} \tilde{p}_{1 \mu} \tilde{p}_{1 \nu}+ \\
V_{3}\left(Q^{2}\right)\left(\tilde{p}_{1 \mu} \widetilde{Q}_{\nu}+\tilde{p}_{1 \nu} \widetilde{Q}_{\mu}\right)+V_{4}\left(Q^{2}\right) \widetilde{Q}_{\mu \nu}+ \\
i V_{5}\left(Q^{2}\right)\left(\tilde{p}_{1 \mu} \widetilde{Q}_{\nu}-\tilde{p}_{1 \nu} \widetilde{Q}_{\mu}\right),
\end{array}
$$


where we introduce the following notations

$$
\begin{gathered}
\widetilde{Q}_{\mu}=Q_{\mu \nu} q_{\nu}-\frac{q_{\mu}}{q^{2}} \bar{Q}, \quad \widetilde{Q}_{\mu} q_{\mu}=0 \\
\widetilde{Q}_{\mu \nu}=Q_{\mu \nu}+\frac{q_{\mu} q_{\nu}}{q^{4}} \bar{Q}-\frac{q_{\nu} q_{\alpha}}{q^{2}} Q_{\mu \alpha}-\frac{q_{\mu} q_{\alpha}}{q^{2}} Q_{\nu \alpha}, \\
\widetilde{Q}_{\mu \nu} q_{\nu}=0, \bar{Q}=Q_{\alpha \beta} q_{\alpha} q_{\beta} .
\end{gathered}
$$

The structure functions $V_{i}\left(Q^{2}\right)(i=1-5)$, which describe the part of the hadronic tensor due to the tensor polarization of the deuteron target, have the following form in terms of the deuteron form factors

$$
\begin{gathered}
V_{1}\left(Q^{2}\right)=-G_{M}^{2}, \quad V_{5}\left(Q^{2}\right)=0, \\
V_{2}\left(Q^{2}\right)=G_{M}^{2}+\frac{4}{1+\eta}\left(G_{C}+\frac{\eta}{3} G_{Q}+\eta G_{M}\right) G_{Q}, \\
V_{3}\left(Q^{2}\right)=-2 \eta\left[G_{M}^{2}+2 G_{Q} G_{M}\right], \\
V_{4}\left(Q^{2}\right)=4 M^{2} \eta(1+\eta) G_{M}^{2}, .
\end{gathered}
$$

The fifth structure function $V_{5}\left(Q^{2}\right)$ is zero since deuteron form factors are real functions in the considered kinematical region. In the time-like region of momentum transfers this structure function is not zero and it is determined by the expression $V_{5}\left(Q^{2}\right)=-4 \eta \operatorname{Im} G_{Q} G_{M}^{*}$ (in this case $Q^{2}$ is the square of the virtual photon four-momentum).

Using the definitions of the cross-section (2) as well as leptonic (3) and hadronic (8) tensors, one can easily derive the expression for the unpolarized differential cross section (in the Born (one-photon-exchange) approximation) in terms of the invariant variables suitable for the calculation of the radiative corrections

$$
\begin{gathered}
\frac{d \sigma_{B}^{u n}}{d Q^{2}}=\frac{\pi \alpha^{2}}{V Q^{4}}\left\{2 \rho W_{1}+\frac{W_{2}}{\tau}[1-\rho(1+\tau)]\right\}, \\
\rho=\frac{Q^{2}}{V}, \quad \tau=\frac{M^{2}}{V} .
\end{gathered}
$$

In the laboratory system this expression can be written in a more familiar form using the standard structure functions $A\left(Q^{2}\right)$ and $B\left(Q^{2}\right)$. Thus, the unpolarized differential cross section for elastic electron-deuteron scattering takes the form

$$
\begin{gathered}
\frac{d \sigma_{B}^{u n}}{d \Omega}=\sigma_{M}\left\{A\left(Q^{2}\right)+B\left(Q^{2}\right) \tan ^{2}\left(\frac{\theta_{e}}{2}\right)\right\}, \\
\sigma_{M}=\frac{\alpha^{2} E^{\prime} \cos ^{2}\left(\frac{\theta_{e}}{2}\right)}{4 E^{3} \sin ^{4}\left(\frac{\theta_{e}}{2}\right)}
\end{gathered}
$$


where $\sigma_{M}$ is the Mott cross section. Here $E$ and $E^{\prime}$ are the incident and scattered electron energies, $\theta_{e}$ is the electron scattering angle

$$
E^{\prime}=E\left[1+2(E / M) \sin ^{2}\left(\theta_{e} / 2\right)\right]^{-1}, Q^{2}=4 E E^{\prime} \sin ^{2}\left(\theta_{e} / 2\right)
$$

The scattering angle in laboratory system can be written in terms of invariants

$$
\cos \theta_{e}=\frac{1-\rho-2 \rho \tau}{1-\rho}, \sin \theta_{e}=\frac{2 \sqrt{\rho \tau(1-\rho-\rho \tau)}}{1-\rho} .
$$

Two structure functions $A\left(Q^{2}\right)$ and $B\left(Q^{2}\right)$ are quadratic combinations of three electromagnetic form factors describing the deuteron structure

$$
\begin{gathered}
A\left(Q^{2}\right)=G_{C}^{2}\left(Q^{2}\right)+\frac{8}{9} \eta^{2} G_{Q}^{2}\left(Q^{2}\right)+\frac{2}{3} \eta G_{M}^{2}\left(Q^{2}\right), \\
B\left(Q^{2}\right)=\frac{4}{3} \eta(1+\eta) G_{M}^{2}\left(Q^{2}\right) .
\end{gathered}
$$

From Eq. (18) one can see that the measurement of the unpolarized cross section at various values of the electron scattering angle and the same value of $Q^{2}$ allows to determine the structure functions $A\left(Q^{2}\right)$ and $B\left(Q^{2}\right)$. Therefore, it is possible to determine the magnetic form factor $G_{M}\left(Q^{2}\right)$ and the following combination of the form factors $G_{C}^{2}\left(Q^{2}\right)+8 \eta^{2} G_{Q}^{2}\left(Q^{2}\right) / 9$. So, the separation of the charge $G_{C}$ and quadrupole $G_{Q}$ form factors requires the polarization measurements.

Before to write similar distributions for the scattering of polarized particles, let us note that for such experimental conditions there may exists, in the general case, the azimuthal correlation between the reaction (electron scattering) plane and the plane $\left(\mathbf{k}_{\mathbf{1}}, \mathbf{s}\right)$ if the initial deuteron is polarized. But in the Born approximation, taking into account the P- and Tinvariance of the hadron electromagnetic interaction, such correlation is absent. Further in this section we consider the situation when the polarization 3-vector $\mathbf{s}$ belongs to the reaction plane and corresponding azimuthal angle equals to zero. Therefore, there exist only two independent components of the polarization vector $\mathbf{s}$ which we call as longitudinal and transverse ones.

To calculate the radiative corrections to the polarization observables it is convenient to parameterize the polarization state of the target (in our case it is the deuteron polarization four-vector $s_{\mu}$ (describing the deuteron vector polarization) and quadrupole polarization tensor $Q_{\mu \nu}$ (describing the deuteron tensor polarization)) in terms of the four-momenta of the 
particles in the reaction under consideration. This parametrization is not unique and depends on the directions along which one defines the longitudinal and transverse components of the deuteron polarization in its rest frame.

As it was mentioned above, we have to define the longitudinal $s^{(L)}$ and transverse $s^{(T)}$ polarization four-vectors. (Often the longitudinal and transverse components of the deuteron polarization are defined along $z$ and $x$ axes). In our case it is naturally to choose the longitudinal direction, in the laboratory system, along the three-momentum transferred $\mathbf{q}$ (the virtual photon momentum) and the transverse direction is perpendicular to the longitudinal one in the reaction plane. The corresponding polarization four-vectors can be written as [34]

$$
\begin{gathered}
s_{\mu}^{(T)}=\frac{(4 \tau+\rho) k_{1 \mu}-(1+2 \tau) q_{\mu}-(2-\rho) p_{1 \mu}}{\sqrt{V c(4 \tau+\rho)}}, \\
s_{\mu}^{(L)}=\frac{2 \tau q_{\mu}-\rho p_{1 \mu}}{M \sqrt{\rho(4 \tau+\rho)}}, c=1-\rho-\rho \tau .
\end{gathered}
$$

These four-vectors satisfy the following conditions: $s^{(L, T)} \cdot p_{1}=0, s^{(L)} \cdot s^{(T)}=0$, and $s^{(L, T) 2}=-1$. So, they have the necessary properties of the polarization four-vectors.

One can verify that the set of the four-vectors $s_{\mu}^{(L, T)}$ in the rest frame of the deuteron (the laboratory system) has the form

$$
\begin{gathered}
s_{\mu}^{(L)}=(0, \mathbf{L}), \quad s_{\mu}^{(T)}=(0, \mathbf{T}), \\
\mathbf{L}=\frac{\mathbf{k}_{\mathbf{1}}-\mathbf{k}_{\mathbf{2}}}{\left|\mathbf{k}_{\mathbf{1}}-\mathbf{k}_{\mathbf{2}}\right|}, \quad \mathbf{T}=\frac{\mathbf{n}_{\mathbf{1}}-\left(\mathbf{n}_{\mathbf{1}} \mathbf{L}\right) \mathbf{L}}{\sqrt{1-\left(\mathbf{n}_{\mathbf{1}} \mathbf{L}\right)^{2}}}, \quad \mathbf{n}_{\mathbf{1}}=\frac{\mathbf{k}_{\mathbf{1}}}{\left|\mathbf{k}_{\mathbf{1}}\right|} .
\end{gathered}
$$

This leads to simple expressions for the spin-dependent hadronic tensors (due to the vector polarization of the deuteron target) corresponding to the longitudinal and transverse direction of the spin four-vector $s_{\mu}$

$$
\begin{gathered}
H_{\mu \nu}^{T}(V)=-\frac{i G_{M} G}{4} \sqrt{\frac{(4 \tau+\rho)}{\tau c}}\left[(4 \tau+\rho)\left(\mu \nu q k_{1}\right)-\right. \\
\left.(2-\rho)\left(\mu \nu q p_{1}\right)\right] \\
H_{\mu \nu}^{L}(V)=\frac{i G_{M}^{2}}{4 \tau}\left(\mu \nu q p_{1}\right) \sqrt{\rho(4 \tau+\rho)}
\end{gathered}
$$

where

$$
G=2 G_{C}+\frac{2}{3} \eta G_{Q}
$$


The spin-dependent parts of the cross-section, due to the vector polarization of the initial deuteron and longitudinal polarization of the electron beam, can be written as

$$
\begin{gathered}
\frac{d \sigma_{B}^{L}}{d Q^{2}}=-\frac{\pi \alpha^{2}}{4 \tau V^{2}} \frac{2-\rho}{\rho} \sqrt{\rho(4 \tau+\rho)} G_{M}^{2}, \\
\frac{d \sigma_{B}^{T}}{d Q^{2}}=-\frac{\pi \alpha^{2}}{V Q^{2}} \sqrt{\frac{(4 \tau+\rho) c}{\tau}} G_{M} G
\end{gathered}
$$

where we assume that $P_{e}$ in Eq.(3) equals to one and the degree of the vector polarization (longitudinal or transverse) of the deuteron target is 100 percent.

In the laboratory system these expressions lead to the following asymmetries (or the spin correlation coefficients) in the elastic electron-deuteron scattering in the Born approximation. These asymmetries are due to the vector polarization of the deuteron target, corresponding to the longitudinal and transverse direction of the spin four-vectors $s_{\mu}^{(L)}$ and $s_{\mu}^{(T)}$, and longitudinal polarization of the electron beam

$$
\begin{gathered}
I_{0} A_{B}^{L}=-\eta \sqrt{(1+\eta)\left(1+\eta \sin ^{2}\left(\frac{\theta_{e}}{2}\right)\right)} \tan \left(\frac{\theta_{e}}{2}\right) \sec \left(\frac{\theta_{e}}{2}\right) G_{M}^{2}, \\
I_{0} A_{B}^{T}=-2 \tan \left(\frac{\theta_{e}}{2}\right) \sqrt{\eta(1+\eta)} G_{M}\left(G_{C}+\frac{\eta}{3} G_{Q}\right),
\end{gathered}
$$

where

$$
I_{0}=A\left(Q^{2}\right)+B\left(Q^{2}\right) \tan ^{2}\left(\frac{\theta_{e}}{2}\right)
$$

It is worth to note that the ratio of the longitudinal polarization asymmetry to the transverse one $A_{B}^{L} / A_{B}^{T}$ is

$$
\frac{A_{B}^{L}}{A_{B}^{T}}=\sqrt{\eta\left(1+\eta \sin ^{2}\left(\frac{\theta_{e}}{2}\right)\right)} \sec \left(\frac{\theta_{e}}{2}\right) \frac{G_{M}}{G} .
$$

This ratio is expressed in terms of the deuteron form factors $G_{M}$ and $G$ in the same way as the corresponding ratio in the case of the elastic electron-proton scattering is expressed via proton electromagnetic form factors $G_{M}^{p}$ and $G_{E}^{p}$, respectively [20, 45]. This is direct consequence of the relation between the proton $H_{\mu \nu}^{p}(V)$ and deuteron $H_{\mu \nu}(V)$ spin-dependent hadronic tensors which depend on the proton polarization and deuteron vector polarization, respectively

$$
H_{\mu \nu}(V)\left(G_{M}, G\right)=-\frac{4 \tau+\rho}{8 \tau} H_{\mu \nu}^{p}(V)\left(G_{M}^{p}, G_{E}^{p}\right) .
$$

Let us consider now the tensor polarized deuteron target. If we introduce for the completeness the orthogonal (to the reaction plane) four-vector $s_{\mu}^{(N)}$ defined as

$$
s_{\mu}^{(N)}=\frac{2 \varepsilon_{\mu \lambda \rho \sigma} p_{1 \lambda} k_{1 \rho} k_{2 \sigma}}{V \sqrt{V c \rho}},
$$


then one can verify that the set of the four-vectors $s_{\mu}^{(I)}, I=L, T, N$ satisfies the conditions

$$
s_{\mu}^{(\alpha)} s_{\mu}^{(\beta)}=-\delta_{\alpha \beta}, \quad s_{\mu}^{(\alpha)} p_{1 \mu}=0, \quad \alpha, \beta=L, T, N
$$

In the rest frame of the deuteron (the laboratory system) the four-vector $s_{\mu}^{(N)}$ has the form

$$
s_{\mu}^{(N)}=(0, \mathbf{N}), \quad \mathbf{N}=\frac{\mathbf{n}_{\mathbf{1}} \times \mathbf{n}_{\mathbf{2}}}{\sqrt{1-\left(\mathbf{n}_{\mathbf{1}} \mathbf{n}_{\mathbf{2}}\right)^{2}}}, \quad \mathbf{n}_{\mathbf{2}}=\frac{\mathbf{k}_{\mathbf{2}}}{\left|\mathbf{k}_{\mathbf{2}}\right|},
$$

then the vector $\mathbf{N}$ is directed along $y$ axis. If to add one more four-vector $s_{\mu}^{(0)}=p_{1 \mu} / M$ to the set of the four-vectors defined by the Eqs. (20) and (29), we receive the complete set of the orthogonal four-vectors with the following properties

$$
s_{\mu}^{(m)} s_{\nu}^{(m)}=g_{\mu \nu}, \quad s_{\mu}^{(m)} s_{\mu}^{(n)}=g_{m n}, \quad m, n=0, L, T, N
$$

This set of the four-vectors allows to express the deuteron quadrupole polarization tensor, in general case, as follows

$$
\begin{gathered}
Q_{\mu \nu}=s_{\mu}^{(m)} s_{\nu}^{(n)} R_{m n} \equiv s_{\mu}^{(\alpha)} s_{\nu}^{(\beta)} R_{\alpha \beta} \\
R_{\alpha \beta}=R_{\beta \alpha}, R_{\alpha \alpha}=0
\end{gathered}
$$

because the time components $R_{00}, R_{0 \alpha}$ and $R_{\alpha 0}$ equal identically to zero due to the condition $Q_{\mu \nu} p_{1 \nu}=0$. Quantities $R_{\alpha \beta}$ are, in fact, the degrees of the tensor polarization of the deuteron target in its rest system (laboratory system). In the Born approximation the components $R_{N L}$ and $R_{N T}$ do not contribute to the observables and this expansion can be rewritten in the following standard form

$$
\begin{gathered}
Q_{\mu \nu}=\left[s_{\mu}^{(L)} s_{\nu}^{(L)}-\frac{1}{2} s_{\mu}^{(T)} s_{\nu}^{(T)}\right] R_{L L}+\frac{1}{2} s_{\mu}^{(T)} s_{\nu}^{(T)}\left(R_{T T}-\right. \\
\left.R_{N N}\right)+\left(s_{\mu}^{(L)} s_{\nu}^{(T)}+s_{\mu}^{(T)} s_{\nu}^{(L)}\right) R_{L T}
\end{gathered}
$$

where we took into account that $R_{L L}+R_{T T}+R_{N N}=0$.

The part of the cross section in the Born approximation that depends on the tensor polarization of the deuteron target can be written as

$$
\frac{d \sigma_{B}^{Q}}{d Q^{2}}=\frac{d \sigma_{B}^{L L}}{d Q^{2}} R_{L L}+\frac{d \sigma_{B}^{T T}}{d Q^{2}}\left(R_{T T}-R_{N N}\right)+\frac{d \sigma_{B}^{L T}}{d Q^{2}} R_{L T},
$$

where

$$
\frac{d \sigma_{B}^{L L}}{d Q^{2}}=\frac{\pi \alpha^{2}}{Q^{4}} 2 c \eta\left\{8 G_{C} G_{Q}+\frac{8}{3} \eta G_{Q}^{2}+\frac{2 c+4 \tau \rho+\rho^{2}}{2 c} G_{M}^{2}\right\}
$$




$$
\begin{gathered}
\frac{d \sigma_{B}^{T T}}{d Q^{2}}=\frac{\pi \alpha^{2}}{Q^{4}} 2 c \eta G_{M}^{2}, \\
\frac{d \sigma_{B}^{L T}}{d Q^{2}}=-\frac{\pi \alpha^{2}}{Q^{4}} 4 \eta(2-\rho) \sqrt{\frac{c \rho}{\tau}} G_{Q} G_{M} .
\end{gathered}
$$

In the laboratory system these expressions lead to the following asymmetries (or analyzing powers) in the elastic electron-deuteron scattering caused by the tensor polarization of the deuteron target and unpolarized electron beam (in the Born approximation)

$$
I_{0} A_{B}^{Q}=A_{B}^{L L} R_{L L}+A_{B}^{T T}\left(R_{T T}-R_{N N}\right)+A_{B}^{L T} R_{L T}
$$

where

$$
\begin{gathered}
I_{0} A_{B}^{L L}=\frac{1}{2}\left\{8 \eta G_{C} G_{Q}+\frac{8}{3} \eta^{2} G_{Q}^{2}+\eta\left[1+2(1+\eta) \tan ^{2}\left(\frac{\theta_{e}}{2}\right)\right] G_{M}^{2}\right\}, \\
I_{0} A_{B}^{T T}=\frac{1}{2} \eta G_{M}^{2}, \quad I_{0} A_{B}^{L T}=-4 \eta \sqrt{\eta+\eta^{2} \sin ^{2}\left(\frac{\theta_{e}}{2}\right)} \sec \left(\frac{\theta_{e}}{2}\right) G_{Q} G_{M} .
\end{gathered}
$$

Using the P-invariance of the hadron electromagnetic interaction, one can parameterize the differential cross section for elastic scattering of longitudinally polarized electron beam on the polarized deuteron target as follows (for the case of the coordinate representation of the deuteron and electron spin-density matrices)

$$
\begin{gathered}
\frac{d \sigma}{d Q^{2}}=\frac{d \sigma^{u n}}{d Q^{2}}\left[1+A^{N} s_{y}+A^{L L} R_{L L}+\right. \\
A^{L T} R_{L T}+A^{T T}\left(R_{T T}-R_{N N}\right)+ \\
\left.P_{e}\left(A^{L} s_{z}+A^{T} s_{x}+A^{L N} R_{L N}+A^{T N} R_{T N}\right)\right],
\end{gathered}
$$

where $d \sigma^{u n} / d Q^{2}$ is the differential cross section for unpolarized particles, $A^{N}$ is the asymmetry (analyzing power) due to the normal component of the deuteron vector polarization $\left(s_{y}\right)$, $A^{L L}, A^{L T}$ and $A^{T T}$ are the asymmetries (analyzing powers) due to the deuteron tensor polarization which correspond to the $R_{L L}, R_{L T}$ and $\left(R_{T T}-R_{N N}\right)$ components of the quadrupole tensor; $A^{L}, A^{T}$ are the correlation parameters due to the longitudinal polarization of the electron beam and $s_{z}, s_{x}$ components of the deuteron vector polarization and $A^{T N}, A^{L N}$ are the correlation parameters due to the longitudinal polarization of the electron beam and $R_{T N}, R_{L N}$ components of the quadrupole tensor. Note that the amplitude of the elastic electron-deuteron scattering is real in the Born (one-photon-exchange) approximation. This fact leads to zero values of the following polarization observables in this approximation: $A^{N}$, $A^{T N}$ and $A^{L N}$. 
The formalism of the spherical tensors is also used for the parametrization of the deuteron spin-density matrix (for the details see Appendix B). In this case the equation (35) can be written as

$$
\begin{gathered}
\frac{d \sigma}{d Q^{2}}=\frac{d \sigma^{u n}}{d Q^{2}}\left[1+2 \operatorname{Imt}_{11} T_{11}+\right. \\
t_{20} T_{20}+2 \operatorname{Ret}_{21} T_{21}+2 \operatorname{Ret}_{22} T_{22}+ \\
\left.P_{e}\left(t_{10} C_{10}+2 \operatorname{Ret}_{11} C_{11}+2 \operatorname{Imt}_{21} C_{21}+2 \operatorname{Imt}_{22} C_{22}\right)\right]
\end{gathered}
$$

where $t_{k q}$ are the polarization tensor describing the polarization state of the deuteron target, $T_{k q}$ and $C_{k q}$ are the analyzing powers and correlation parameters of the reaction, respectively.

The relations between the polarization observables in the coordinate representation and approach of the spherical tensors are the following

$$
\begin{gathered}
T_{11}=-\frac{1}{\sqrt{3}} A_{y}, T_{20}=-\frac{\sqrt{2}}{3} A_{z z}, T_{21}=\frac{1}{2 \sqrt{3}} A_{x z}, \\
T_{22}=-\frac{1}{\sqrt{3}} A_{x x}, C_{10}=\sqrt{\frac{2}{3}} A_{z}, C_{11}=-\frac{1}{\sqrt{3}} A_{x} \\
C_{21}=\frac{1}{2 \sqrt{3}} A_{y z}, C_{22}=-\frac{1}{2 \sqrt{3}} A_{x y} .
\end{gathered}
$$

If the longitudinal direction is determined by the recoil deuteron three-momentum, the relations (21) do not affected by hard photon radiation in the lepton part of interaction (this corresponds to use of the so-called hadronic variables) because $\mathbf{q}=\mathbf{p}_{\mathbf{2}}-\mathbf{p}_{\mathbf{1}}$. But when this direction is reconstructed from the experiment using the three-momentum of the detected scattered electron (lepton variables), these relations break down because $\mathbf{q} \neq \mathbf{k}_{\mathbf{1}}-\mathbf{k}_{\mathbf{2}}$ in this case. It means that in the leptonic variables the parametrization (20) is unstable and radiation of hard photon by electron leads to mixture of the longitudinal and transverse polarizations.

One can get rid of such mixture if we choose, in the laboratory system of the reaction (1), the longitudinal direction $\mathbf{l}$ along the electron beam momentum and the transverse one $\mathbf{t}$ - in the plane $\left(\mathbf{k}_{\mathbf{1}}, \mathbf{k}_{\mathbf{2}}\right)$ and perpendicular to $\mathbf{l}$. Then the corresponding parametrization of the polarization four-vectors is [34]

$$
\begin{gathered}
s_{\mu}^{(l)}=\frac{2 \tau k_{1 \mu}-p_{1 \mu}}{M}, \quad s_{\mu}^{(n)}=s_{\mu}^{(N)}, \\
s_{\mu}^{(t)}=\frac{k_{2 \mu}-(1-\rho-2 \rho \tau) k_{1 \mu}-\rho p_{1 \mu}}{\sqrt{V c \rho}} .
\end{gathered}
$$


One can verify that the set of these polarization four-vectors $s_{\mu}^{(l, t, n)}$ in the rest frame of the deuteron (the laboratory system) has the form

$$
\begin{gathered}
s_{\mu}^{(l)}=(0, \mathbf{l}), \quad s_{\mu}^{(t)}=(0, \mathbf{t}), \quad s_{\mu}^{(n)}=(0, \mathbf{n}) \\
\mathbf{l}=\mathbf{n}_{\mathbf{1}}, \quad \mathbf{t}=\frac{\mathbf{n}_{\mathbf{2}}-\left(\mathbf{n}_{\mathbf{1}} \mathbf{n}_{\mathbf{2}}\right) \mathbf{n}_{\mathbf{1}}}{\sqrt{1-\left(\mathbf{n}_{\mathbf{1}} \mathbf{n}_{\mathbf{2}}\right)^{2}}}, \quad \mathbf{n}=\frac{\mathbf{n}_{\mathbf{1}} \times \mathbf{n}_{\mathbf{2}}}{\sqrt{1-\left(\mathbf{n}_{\mathbf{1}} \mathbf{n}_{\mathbf{2}}\right)^{2}}}
\end{gathered}
$$

And this set of the polarization four-vectors (together with four-vector $s_{\mu}^{(0)}$ ) is also a complete set of orthogonal four-vectors with the properties

$$
s_{\mu}^{(m)} s_{\nu}^{(m)}=g_{\mu \nu}, \quad s_{\mu}^{(m)} s_{\mu}^{(n)}=g_{m n}, \quad m, n=0, l, t, n .
$$

Hadronic tensors $H_{\mu \nu}^{l, t}$ corresponding to the longitudinal and transverse directions of the new spin four-vectors have the following form

$$
\begin{gathered}
H_{\mu \nu}^{l}=i \frac{4 \tau+\rho}{4 \tau}\left\{G\left[-2 \tau\left(\mu \nu q k_{1}\right)+\frac{2 \tau(2-\rho)}{4 \tau+\rho}\left(\mu \nu q p_{1}\right)\right]+G_{M} \frac{\rho(1+2 \tau)}{4 \tau+\rho}\left(\mu \nu q p_{1}\right)\right\} G_{M} \\
H_{\mu \nu}^{t}=i \sqrt{\frac{\rho \tau}{c}}\left\{G(1+2 \tau)\left[\frac{2-\rho}{4 \tau}\left(\mu \nu q p_{1}\right)-\frac{4 \tau+\rho}{4 \tau}\left(\mu \nu q k_{1}\right)\right]-G_{M} \frac{c}{2 \tau}\left(\mu \nu q p_{1}\right)\right\} G_{M} .
\end{gathered}
$$

In the case of the scattering off vector-polarized deuteron target the tensors $H_{\mu \nu}^{L, T}$ and $H_{\mu \nu}^{l, t}$ corresponding to two choices of the spin four-vectors are connected by trivial relations

$$
H_{\mu \nu}^{L}=\cos \theta H_{\mu \nu}^{l}+\sin \theta H_{\mu \nu}^{t}, H_{\mu \nu}^{T}=-\sin \theta H_{\mu \nu}^{l}+\cos \theta H_{\mu \nu}^{t},
$$

where

$$
\cos \theta=-\left(s^{(L)} s^{(l)}\right), \sin \theta=-\left(s^{(L)} s^{(t)}\right) .
$$

The simple calculation leads to

$$
\cos \theta=\frac{\rho(1+2 \tau)}{\sqrt{\rho(4 \tau+\rho)}}, \quad \sin \theta=-2 \sqrt{\frac{c \tau}{4 \tau+\rho}} .
$$

These relations are the consequence of the fact that two sets of the spin four-vectors are connected by means of orthogonal matrix which describes the rotation in the plane perpendicular to the direction $\mathbf{n}=\mathbf{N}$

$$
s_{\mu}^{(A)}=V_{A \beta}(\theta) s_{\mu}^{(\beta)}, V(\theta)=\left(\begin{array}{cc}
\cos \theta & \sin \theta \\
-\sin \theta & \cos \theta
\end{array}\right),
$$

where $A=L, T, \beta=l, t$. 
Using this rotation matrix one can write the spin-dependent parts (due to the vector polarization of the target) of the Born cross-section, which correspond to parametrization (38), in the simple way

$$
\frac{d \sigma_{B}^{\beta}}{d Q^{2}}=V_{\beta A}(-\theta) \frac{d \sigma_{B}^{A}}{d Q^{2}},
$$

where the quantities $d \sigma_{B}^{L} / d Q^{2}$ and $d \sigma_{B}^{T} / d Q^{2}$ are defined by Eqs.(23) and (24). Therefore, we can write

$$
\begin{gathered}
\frac{d \sigma_{B}^{l}}{d Q^{2}}=-\frac{\pi \alpha^{2}}{V^{2}}\left[\frac{1+2 \tau}{4 \tau}(2-\rho) G_{M}+\frac{2 c}{\rho} G\right] G_{M}, \\
\frac{d \sigma_{B}^{t}}{d Q^{2}}=\frac{\pi \alpha^{2}}{V Q^{2}} \sqrt{\frac{c \rho}{\tau}}\left[\frac{1}{2}(2-\rho) G_{M}-(1+2 \tau) G\right] G_{M} .
\end{gathered}
$$

In the case of the tensor polarization of the deuteron target, the relations which are an analogue of Eq.(43) read

$$
\frac{d \sigma_{B}^{\beta}}{d Q^{2}}=T_{\beta A}(-\theta) \frac{d \sigma_{B}^{A}}{d Q^{2}},
$$

where now $A=L L, T T, L T ; \beta=l l$, tt, lt, respectively. The rotation matrix in this case can be written as

$$
T(\theta)=\left(\begin{array}{ccc}
\frac{1}{4}(1+3 \cos 2 \theta) & \frac{3}{4}(1-\cos 2 \theta) & \frac{3}{4} \sin 2 \theta \\
\frac{1}{4}(1-\cos 2 \theta) & \frac{1}{4}(3+\cos 2 \theta) & \frac{-1}{4} \sin 2 \theta \\
-\sin 2 \theta & \sin 2 \theta & \cos 2 \theta
\end{array}\right)
$$

where the partial cross sections $d \sigma_{B}^{I J} / d Q^{2}, I, J=L, T$ are defined in Eq.(32) as the coefficients in front of the quantities $R_{L L}, R_{T T}-R_{N N}$ and $R_{L N}$, respectively, and the partial cross sections in the left hand side of Eq.(46) are defined as follows

$$
\frac{d \sigma_{B}^{Q}}{d Q^{2}}=\frac{d \sigma_{B}^{l l}}{d Q^{2}} R_{l l}+\frac{d \sigma_{B}^{t t}}{d Q^{2}}\left(R_{t t}-R_{n n}\right)+\frac{d \sigma_{B}^{l t}}{d Q^{2}} R_{l t} .
$$

The partial spin-dependent cross sections in this case are

$$
\begin{gathered}
\frac{d \sigma_{B}^{t t}}{d Q^{2}}=\frac{2 \pi \alpha^{2}}{Q^{4}} c \eta\left[(1+\tau \rho) G_{M}^{2}+\frac{2 \rho}{\rho+4 \tau}(2-\rho)(1+2 \tau) G_{M} G_{Q}+\frac{2 c}{1+\eta} G_{Q} G\right] \\
\frac{d \sigma_{B}^{l t}}{d Q^{2}}=-\frac{8 \pi \alpha^{2}}{Q^{4}} \eta \sqrt{c \eta}\left\{\frac{1+2 \tau}{1+\eta}\left[\rho \tau(1+\eta) G_{M}^{2}+2 c G_{Q} G\right]+2\left[2 \rho \frac{1+2 \tau}{\rho+4 \tau}(c+\tau+\tau \eta)-c\right] G_{M} G_{Q}\right\} \\
\frac{d \sigma_{B}^{l l}}{d Q^{2}}=-\frac{2 \pi \alpha^{2}}{Q^{4}} \eta\left\{\left[\frac{\rho}{2}(2+4 \tau-\rho)-1-3 \rho^{2} \tau(1+\tau)\right] G_{M}^{2}+6 c \rho(2-\rho)\right. \\
\left.\frac{1+2 \tau}{\rho+4 \tau} G_{M} G_{Q}+\frac{c}{\tau(1+\eta)}[2 \tau-\rho-6 \rho \tau(1+\tau)] G_{Q} G\right\}
\end{gathered}
$$


As one can see, now the spin-dependent part of the cross section, due to the tensor polarization of the deuteron target, is expressed in terms of new polarization parameters $R_{l l}, R_{t t}-R_{n n}$ and $R_{l t}$ which are defined in accordance with the new longitudinal and transverse directions given by Eq.(38), and the coefficients in front of these quantities, in the right side of Eq.(47), define the corresponding partial cross sections $d \sigma_{B}^{i j} / d Q^{2}$. The new polarization parameters are related to $R_{L L}, R_{T T}-R_{N N}$ and $R_{L T}$

$$
\begin{gathered}
R_{l l}=\frac{1}{4}(1+3 \cos 2 \theta) R_{L L}+\frac{1}{4}(1-\cos 2 \theta)\left(R_{T T}-R_{N N}\right)-\sin 2 \theta R_{L T}, \\
R_{l l}-R_{n n}=\frac{3}{4}(1-\cos 2 \theta) R_{L L}+\frac{1}{4}(3+\cos 2 \theta)\left(R_{T T}-R_{N N}\right)+\sin 2 \theta R_{L T}, \\
R_{l t}=\frac{3}{4} \sin 2 \theta R_{L L}-\frac{1}{4} \sin 2 \theta\left(R_{T T}-R_{N N}\right)+\cos 2 \theta R_{L T} .
\end{gathered}
$$

Consider now the scattering of the longitudinally polarized electron beam by the unpolarized deuteron target provided that the recoil deuteron is polarized. In this case we can calculate both the vector and tensor polarizations of the recoil deuteron by means of the results given above. To do this note that the polarization state of the recoil deuteron can be described by the longitudinal and transverse polarization four-vectors $S_{\mu}^{(L)}$ and $S_{\mu}^{(T)}$, which satisfied relations $S^{2}=-1, S \cdot p_{2}=0$, and they are

$$
S_{\mu}^{(L)}=\frac{2 \tau q_{\mu}+\rho p_{2 \mu}}{M \sqrt{\rho(4 \tau+\rho)}}, \quad S_{\mu}^{(T)}=s_{\mu}^{(T)} .
$$

Note that the spin-dependent part of the hadronic tensor describing the vector polarization of the deuteron target, Eq.(12), can be written in the following equivalent form

$$
\begin{aligned}
H_{\mu \nu}(V)= & \frac{i G_{M}}{2 M}\left[\left(G_{M}-G\right) s \cdot p_{2}\left(\mu \nu q p_{1}\right)+\right. \\
& \left.2 M^{2}(1+\eta) G(\mu \nu q s)\right] .
\end{aligned}
$$

The spin-dependent part of the hadronic tensor $H_{\mu \nu}^{R}(V)$, which corresponds to the case of the vector polarized recoil deuteron, can be derived from this equation by following substitution: $s_{\mu} \rightarrow S_{\mu}, p_{1} \leftrightarrow-p_{2}$. In fact this means that we have to change the term $s \cdot p_{2}$ in the right side of Eq. (50) by the term $S \cdot p_{1}$. The vector polarization of the recoil deuteron (longitudinal $P^{L}$ or transverse $P^{T}$ ) is defined as a ratio of the spin-dependent part of the cross section to the unpolarized one. Taking into account that $S^{(L)} \cdot p_{1}=-s^{(L)} \cdot p_{2}$ and $s^{(T)} \cdot q=0$ we conclude that

$$
P^{L}=-A^{L}, \quad P^{T}=A^{T},
$$


where $A^{L}$ and $A^{T}$ are the respective vector asymmetries for the scattering of the longitudinally polarized electron beam by the vector polarized deuteron target (we assume that beam and target have 100 percent polarization).

Here we want to pay attention to the fact that determination of the ratio $G_{M} / G$ by means of the measurement of the ratio of the asymmetries $A^{L} / A^{T}$, in the scattering of the longitudinally polarized electron beam by the vector polarized deuteron target, may be more attractive than by measuring the ratio of the polarizations $P^{L} / P^{T}$, in the polarization transfer (from the longitudinally polarized electron beam to the recoil deuteron) process, because in the last case the second scattering is necessary. This decreases the corresponding events number for about two orders [46] and increasing essentially the statistical error. The problem with depolarization effect that appears in the scattering of high-intensity electron beam on the polarized solid target can be avoided using the polarized gas deuteron target [13].

By analogy, the components of the tensor polarization of the recoil deuteron are defined by the ratios of the corresponding partial spin-dependent cross sections to the unpolarized one

$$
\widetilde{R}_{L L}=\frac{d \sigma_{B}^{L L}}{d \sigma_{B}^{u n}}, \widetilde{R}_{L T}=\frac{d \sigma_{B}^{L T}}{d \sigma_{B}^{u n}}, \widetilde{R}_{T T}-\widetilde{R}_{N N}=\frac{d \sigma_{B}^{T T}}{d \sigma_{B}^{u n}}
$$

The spin-dependent part of the hadronic tensor $H_{\mu \nu}^{R}(T)$, which corresponds to the case of the tensor polarized recoil deuteron, can be derived from Eq.(14) by changing sign of the structure function $V_{3}\left(Q^{2}\right)$. The straightforward calculations using this updated tensor and parametrization (49) leads to the following results: i) both diagonal partial cross sections in the right-hand side of the last equations are the same ones as defined by the first and second line in Eqs.(32) for the scattering off the polarized target, ii) partial cross section $d \sigma_{B}^{L T} / d Q^{2}$ changes sign as compared with the one given by the third line in Eqs.(32).

\section{RADIATIVE CORRECTIONS}

There exist two sources of the radiative corrections when we take into account the corrections of the order of $\alpha$. The first one is caused by virtual and soft photon emission that cannot affect the kinematics of the process (1). The second one arises due to the radiation of a hard photon

$$
e^{-}\left(k_{1}\right)+D\left(p_{1}\right) \rightarrow e^{-}\left(k_{2}\right)+\gamma(k)+D\left(p_{2}\right)
$$


because cuts on the event selection used in the current experiments allow to radiate photons with the energy about $100 \mathrm{MeV}$ and even more [16,46]. Such photons cannot be interpreted as "soft" ones. The form of the radiative corrections caused by the contribution due to the hard photon emission depends strongly on the choice of the variables which are used to describe process (52) [47].

We calculate the radiative corrections in the leptonic variables. This corresponds to the experimental setup when the energy and momentum of the virtual photon is determined with the help of the measured energy and momentum of the scattered electron.

The hadronic variables (in this case the reconstruction of the virtual photon kinematics is done using the recoil deuteron energy and momentum) were used formerly to compute the radiative corrections in the elastic and deep-inelastic polarized electron-proton scattering [47, 48], and elastic polarized electron-deuteron scattering [35].

We calculate here the model-independent radiative corrections which includes all QED corrections to the lepton part of the interaction and insertion of the vacuum polarization into the exchanged virtual photon propagator.

The general analysis of the two-photon exchange (box diagrams) contribution to the polarization observables in the elastic electron-deuteron scattering was done in the Refs. [49, 50]. The numerical estimation of the two-photon exchange effect on the deuteron electromagnetic form factors was given in the Ref. [51].

\subsection{Unpolarized cross section}

The model-independent radiative corrections to the unpolarized and polarized (due to the vector polarization of the deuteron target) cross sections of the elastic electron-deuteron scattering can be obtained using the results of the paper [45] where the QED corrections for the polarized elastic electron-proton scattering were calculated in the framework of the electron structure functions.

The spin-independent part of the cross section for the elastic electron-deuteron scattering can be derived from the respective part of the elastic electron-proton scattering by a simple rule using following relation between spin-independent hadronic tensors describing electrondeuteron and electron-proton scattering

$$
H_{\mu \nu}^{d(u n)}=\frac{4 \tau+x y r}{4 \tau} H_{\mu \nu}^{p(u n)}\left(G_{M p}^{2} \rightarrow \frac{2}{3} G_{M}^{2}\right.
$$




$$
\left.G_{E p}^{2} \rightarrow G_{C}^{2}+\frac{x^{2} y^{2} r^{2}}{18 \tau^{2}} G_{Q}^{2}\right)
$$

where the variables $x, y$ and $r$ are defined as follows

$$
\begin{gathered}
x=\frac{Q^{2}}{2 p_{1}\left(k_{1}-k_{2}\right)}, \quad y=\frac{2 p_{1}\left(k_{1}-k_{2}\right)}{V}, \\
r=\frac{-\left(k_{1}-k_{2}-k\right)^{2}}{Q^{2}} .
\end{gathered}
$$

Remember that $k$ is the four-momentum of the hard photon in the reaction (52).

The radiatively corrected cross section can be written by means of the electron structure functions in the following form (master formula)[52]

$$
\begin{gathered}
\frac{d \sigma\left(k_{1}, k_{2}\right)}{d Q^{2}}=\int_{y_{\min }}^{y_{\max }} d y \int_{z_{1 m}}^{1} d z_{1} \int_{z_{2 m}}^{1} d z_{2} D\left(z_{1}, L\right) . \\
\frac{1}{z_{2}^{2}} D\left(z_{2}, L\right) \frac{d \sigma_{\text {hard }}\left(\tilde{k}_{1}, \tilde{k}_{2}\right)}{d \tilde{Q}^{2} d \tilde{y}}, \quad L=\ln \frac{Q^{2}}{m^{2}},
\end{gathered}
$$

where $m$ is the electron mass and the limits of the integration with respect to the variables $y, z_{1}$ and $z_{2}$ are defined below, the quantity $D(z, L)$ is the electron structure function. The numerical estimations of the radiative corrections (see below) have been done with the help of the exponentiation form of the electron structure function which is given in Ref. [45, 53]. For the different representations of the photon contribution to the electron structure function see, for example, Ref. [54].

The reduced variables which define the cross section with emission of the hard photon in the integrand are

$$
\tilde{k}_{1}=z_{1} k_{1}, \tilde{k}_{2}=\frac{k_{2}}{z_{2}}, \widetilde{Q}^{2}=\frac{z_{1}}{z_{2}} Q^{2}, \tilde{y}=1-\frac{1-y}{z_{1} z_{2}}
$$

The hard part of unpolarized (spin-independent) cross section can be written as follow

$$
\frac{d \sigma_{h a r d}}{d Q^{2} d y}=\frac{d \sigma_{B}^{u n}}{d Q^{2} d y}\left(1+\frac{\alpha}{2 \pi} \delta(x, \rho)\right)+H_{x}+H_{x r}
$$

where

$$
\delta(x, \rho)=-1-\frac{\pi^{2}}{3}-2 f\left(\frac{x-\rho(1+x \tau)}{x(1-\rho)\left(1-z_{+}\right)}\right)-\ln ^{2} \frac{(1-\rho)}{1-z_{+}}, \quad f(x)=\int_{0}^{x} \frac{d t}{t} \ln (1-t)
$$




$$
\begin{gathered}
H_{x}=\frac{\alpha}{V^{2}}\left[\frac{1-r_{1}}{1-\rho} \hat{P}_{1}-\frac{1-r_{2}}{1-z_{+}} \hat{P}_{2}\right] N(x, r) \frac{\alpha^{2}\left(r Q^{2}\right)}{r}, \\
H_{x r}=\frac{\alpha}{V^{2}}\left\{\int_{r_{-}}^{r_{+}} \frac{2 x W(x, r) d r}{\sqrt{\rho^{2}+4 \rho x^{2} \tau}}+P \int_{r_{-}}^{r_{+}} \frac{d r}{1-r}\left[\frac { 1 - \hat { P } _ { 1 } } { | r - r _ { 1 } | } \left(\frac{1+r^{2}}{1-\rho} N(x, r)+\right.\right.\right. \\
\left.\left.\left.+\left(r_{1}-r\right) T_{1}(x, r)\right)-\frac{1-\hat{P}_{2}}{\left|r-r_{2}\right|}\left(\frac{1+r^{2}}{1-z_{+}} N(x, r)+\left(r_{2}-r\right) T_{2}(x, r)\right)\right]\right\} \frac{\alpha^{2}\left(r Q^{2}\right)}{r}, \\
N(x, r)=\frac{2}{3}\left(1+\lambda_{r}\right) G_{M}^{2}\left(r Q^{2}\right)+\frac{2}{\rho^{2} r}\left(1-\frac{\rho}{x}-\rho \tau\right) G^{2}\left(\lambda_{r}, r Q^{2}\right), \\
T_{1}(x, r)=-\frac{2}{\rho^{2} r}\left[1-\frac{2(x-\rho)}{x}\right] G^{2}\left(\lambda_{r}, r Q^{2}\right), T_{2}(x, r)=-\frac{2}{\rho^{2} r}\left[1-r-\frac{\rho}{x}\right] G^{2}\left(\lambda_{r}, r Q^{2}\right), \\
\lambda_{r}=\frac{\rho r}{4 \tau}, G^{2}\left(\lambda_{r}, r Q^{2}\right)=G_{C}^{2}\left(r Q^{2}\right)+\frac{2}{3} \lambda_{r} G_{M}^{2}\left(r Q^{2}\right)+\frac{8}{9} \lambda_{r}^{2} G_{Q}^{2}\left(r Q^{2}\right), z_{+}=\frac{\rho}{x}(1-x) .
\end{gathered}
$$

The limits of integration with respect to the variable $r$ in expression $H_{x r}$ can be written as

$$
r_{ \pm}=\frac{1}{2 x^{2}\left(\tau+z_{+}\right)}\left[2 x^{2} \tau+(1-x)\left(\rho \pm \sqrt{\rho^{2}+4 x^{2} \rho \tau}\right)\right] .
$$

The limits of integration in the master formula (55) at fixed values of $\rho$ can be derived from the restriction on the lost invariant mass for the hard subprocess:

$$
M^{2}<\left(\tilde{k}_{1}+p_{1}-\tilde{k}_{2}\right)^{2}<\left(M+\Delta_{M}\right)^{2}
$$

where usually $\Delta_{M}$ is smaller than the pion mass to exclude inelastic hadronic events. It means that

$$
\begin{gathered}
z_{2 m}=\rho+\frac{1-y}{z_{1}}, \quad z_{1 m}=\frac{1-y}{1-\rho}, \quad y_{\min }=\rho, \\
y_{\max }=\rho+\Delta_{t h}, \quad \Delta_{t h}=\left(\Delta_{M}^{2}+2 M \Delta_{M}\right) / V .
\end{gathered}
$$

The action of the projection operators $\hat{P}_{1}$ and $\hat{P}_{2}$ is defined as follows

$$
\hat{P}_{1} f(r, x)=f\left(r_{1}, x\right), \quad \hat{P}_{2} f(r, x)=f\left(r_{2}, x\right),
$$

where

$$
r_{1}=\frac{x-\rho}{x(1-\rho)}, \quad r_{2}=\frac{x}{x-\rho(1-x)}=\frac{1}{1-z_{+}} .
$$


The symbol of the principal value $P$ in expression for $H_{x r}$ means that one has to ignore nonphysical singularity at $r=1$, other words

$$
\begin{gathered}
P \int_{r_{-}}^{r_{+}} \frac{f(r) d r}{(1-r)\left|r-r_{1}\right|}=\int_{r_{-}}^{r_{+}} \frac{d r}{(1-r)}\left[\frac{f(r)}{\left|r-r_{1}\right|}-\right. \\
\left.\frac{f(1)}{\left|1-r_{1}\right|}\right]+\frac{f(1)}{\left|1-r_{1}\right|} \ln \frac{1-r_{-}}{r_{+}-1} .
\end{gathered}
$$

The Born unpolarized cross section that enters into hard cross-section (56) is defined by expression (17) multiplied by delta function $\delta(y-\rho)$.

To compare our calculations with other ones it is very important to extract the first order correction from the master formula. For this goal it is enough to use well known iterative form of the electron structure function entering Eq.(55) taking into account terms of the order of $\alpha$, namely

$$
\begin{gathered}
D\left(z_{i}, L\right)=\lim _{\Delta_{i} \rightarrow 0}\left[\delta\left(1-z_{i}\right)+\frac{\alpha(L-1)}{2 \pi} P_{1}\left(z_{i}\right)\right], \\
P_{1}(z)=\frac{1+z^{2}}{1-z} \Theta\left(1-z-\Delta_{i}\right)+\delta(1-z)\left(\frac{3}{2}+2 \ln \Delta_{i}\right) .
\end{gathered}
$$

The exact form of the infrared parameters $\Delta_{1}$ and $\Delta_{2}$ is given in Ref. [45] but it is unessential because they cancel in the final result that can be written as follows

$$
\begin{gathered}
\frac{d \sigma}{d Q^{2}}=\frac{d \sigma_{B}}{d Q^{2}}\left[1+\frac{\alpha}{2 \pi}\left(\delta(1, \rho)+(L-1) G_{0}\right)\right] \\
+\frac{\alpha}{2 \pi}(L-1) G_{1}+\int_{y_{\min }}^{y_{\max }}\left(H_{x}+H_{x r}\right) d y
\end{gathered}
$$

where

$$
\begin{gathered}
G_{0}=g(z)+g(\tilde{z}), \quad G_{1}=I(z)+\tilde{I}(\tilde{z}), z=\frac{\Delta_{t h}}{1-\rho} \\
g(x)=\frac{3}{2}-2 x+\frac{x^{2}}{2}+2 \ln x, \tilde{z}=\Delta_{t h} \\
I(z)=\int_{1-z}^{1} \frac{1+z_{1}^{2}}{1-z_{1}}\left[\frac{d \sigma_{B}\left(z_{1} k_{1}, k_{2}\right)}{d Q^{2}}-\frac{d \sigma_{B}\left(k_{1}, k_{2}\right)}{d Q^{2}}\right] d z_{1}
\end{gathered}
$$

and $\tilde{I}$ can be derived from $I$ by substitution $d \sigma_{B}\left(k_{1}, z_{2}^{-1} k_{2}\right)$ instead of $d \sigma_{B}\left(z_{1} k_{1}, k_{2}\right)$ and $z_{1} \rightarrow z_{2}$. 
3.2. Correction to the part of the cross section caused by the vector polarization of the deuteron target

The correction to the spin-dependent part of the cross section, provided that the deuteron target has vector polarization, can be obtained from corresponding formulas of the electronproton scattering by full analogy with unpolarized case. The only difference consists in new connection between spin-dependent hadronic tensors that reads

$$
\begin{gathered}
H_{\mu \nu}^{d(l, t)}=-\frac{4 \tau+x y r}{8 \tau} H_{\mu \nu}^{p(l, t)}\left(G_{M p} \rightarrow G_{M},\right. \\
\left.G_{E p} \rightarrow 2 G_{C}+\frac{x y r}{6 \tau} G_{Q}\right) .
\end{gathered}
$$

We again can start from the Drell-Yan representation but now for the spin-dependent part of the cross section. Remind that this representation is valid in this case if the radiation of collinear photons by the initial and final electrons do not change longitudinal $(l)$ and transverse $(t)$ polarizations. Such stabilize polarization four-vectors of the deuteron polarization can be written in the form

$$
\begin{gathered}
\widetilde{S}_{\mu}^{(l)}=\frac{2 \tau k_{1 \mu}-p_{1 \mu}}{M} \\
\widetilde{S}_{\mu}^{(t)}=\frac{-x y p_{1 \mu}+k_{2 \mu}-[-2 x y \tau+(1-y)] k_{1 \mu}}{\sqrt{V x y(1-y-x y \tau)}} .
\end{gathered}
$$

One can verify that the polarization four-vector $\widetilde{S}^{(l)}$ in the laboratory system has components $(0, \vec{n})$, where three-vector $\vec{n}$ has orientation of the initial electron three-momentum $\vec{k}_{1}$. One can verify also that $\tilde{S}^{(t)} \widetilde{S}^{(l)}=0$ and in the laboratory system

$$
\widetilde{S}^{(t)}=\left(0, \mathbf{n}_{\perp}\right), \mathbf{n}_{\perp}^{2}=1, \quad \mathbf{n n}_{\perp}=0
$$

where three-vector $\mathbf{n}_{\perp}$ belongs to the plane $\left(\mathbf{k}_{\mathbf{1}}, \mathbf{k}_{\mathbf{2}}\right)$.

It is convenient to write down the master formula for the spin-dependent differential cross sections $d \sigma^{l}$ and $d \sigma^{t}$ in the form

$$
\begin{gathered}
\frac{d \sigma^{l, t}\left(k_{1}, k_{2}, \widetilde{S}\right)}{d Q^{2}}=\int_{y_{\min }}^{y_{\max }} d y \frac{d \sigma^{l, t}}{d Q^{2} d y}, \\
\frac{d \sigma^{l, t}}{d Q^{2} d y}=\int_{z_{1 m}}^{1} d z_{1} \int_{z_{2 m}}^{1} \frac{d z_{2}}{z_{2}^{2}} D^{(p)}\left(z_{1}, L\right) \bullet
\end{gathered}
$$




$$
D\left(z_{2}, L\right) \frac{d \sigma_{h a r d}^{l, t}\left(\tilde{k}_{1}, \tilde{k}_{2}, \widetilde{S}\right)}{d \tilde{Q}^{2} d \tilde{y}}
$$

Function $D^{(p)}(z, L)$ is the electron structure function for the case of the longitudinally polarized electron. It differs from $D(z, L)$ yet in the second order due to collinear electron-positron pair production in the so-called nonsinglet channel (for the details see Ref. [55]).

If the longitudinal direction $\mathbf{L}$ is chosen along the three-momentum $\mathbf{k}_{\mathbf{1}}-\mathbf{k}_{\mathbf{2}}$ in the laboratory system, which for non-radiative process coincides with direction of the three-vector $\mathbf{q}$, and the transverse one $\mathbf{T}$ lies in the plane $\left(\mathbf{k}_{\mathbf{1}}, \mathbf{k}_{\mathbf{2}}\right)$, one has to use

$$
\begin{gathered}
S_{\mu}^{(L)}=\frac{2 \tau\left(k_{1}-k_{2}\right)_{\mu}-y p_{1 \mu}}{M \sqrt{y(y+4 x \tau)}} \\
S_{\mu}^{(T)}=\frac{(1+2 x \tau) k_{2 \mu}-(1-y-2 x \tau) k_{1 \mu}-x(2-y) p_{1 \mu}}{\sqrt{V x(1-y-x y \tau)(y+4 x \tau)}} .
\end{gathered}
$$

In this case we use relations

$$
\frac{d \sigma^{(A)}\left(k_{1}, k_{2}, S\right)}{d Q^{2}}=\int_{y_{\min }}^{y_{\max }} d y V_{A \beta}(\psi) \frac{d \sigma^{\beta}\left(k_{1}, k_{2}, \widetilde{S}\right)}{d Q^{2} d y}
$$

where by the full analogue with Eq.(42)

$$
\begin{gathered}
\cos \psi=-\left(S^{(L)} \tilde{S}^{(l)}\right), \sin \psi=-\left(S^{(L)} \tilde{S}^{(t)}\right) \\
\cos \psi=\frac{y+2 x y \tau}{\sqrt{y^{2}+4 x y \tau}}, \quad \sin \psi=-2 \sqrt{\frac{x y \tau(1-y-x y \tau)}{y^{2}+4 x y \tau}} .
\end{gathered}
$$

The vector asymmetries in the considered process with accounting radiative corrections are defined as a following ratios

$$
A_{C}^{l, t}=\frac{d \sigma^{l, t}\left(k_{1}, k_{2}, \widetilde{S}\right)}{d \sigma\left(k_{1}, k_{2}\right)}, A_{C}^{L, T}=\frac{d \sigma^{L, T}\left(k_{1}, k_{2}, S\right)}{d \sigma\left(k_{1}, k_{2}\right)}
$$

where the unpolarized cross-section is described by Eq.(55), and the partial cross-sections caused by correlation between the deuteron vector polarizations and the electron longitudinal polarization (we use $P_{e}=1$ ) are defined by Eqs. (64) and (65), respectively. Therefore, the calculation of the asymmetry, including the radiative corrections, requires the knowledge of the radiative corrections for both spin-independent and spin-dependent parts of the cross section.

The hard part of the polarized cross section which enter under the integral sign in the right-hand side of Eq.(64) can be written in the very similar, but slightly different, form as 
compared with unpolarized case

$$
\begin{gathered}
\frac{d \sigma_{\text {hard }}^{l, t}}{d Q^{2} d y}=\frac{d \sigma_{B}^{l, t}}{d Q^{2} d y}\left(1+\frac{\alpha}{2 \pi} \delta(x, \rho)\right)+H_{x}^{l, t}+H_{x r}^{l, t}, \\
H_{x}^{l, t}=-\frac{\alpha U^{l, t}}{8 \tau V^{2}}\left\{-\frac{1-r_{1}}{1-\rho} \hat{P}_{1} N_{1}^{l, t}-\frac{1-r_{2}}{1-z_{+}} \hat{P}_{2} N_{2}^{l, t}\right\} \frac{\alpha^{2}\left(r Q^{2}\right)}{r}, \\
H_{x r}^{l, t}=-\frac{\alpha U^{l, t}}{8 \tau V^{2}}\left\{\int_{r_{-}}^{r_{+}} \frac{2 x W^{l, t} d r}{\sqrt{\rho^{2}+4 x^{2} \rho \tau}}+P \int_{r_{-}}^{r_{+}} \frac{d r}{1-r}\left[\frac{1-\hat{P}_{1}}{\left|r-r_{1}\right|}\left(\frac{1+r^{2}}{1-\rho} N_{1}^{l, t}+\left(r_{1}-r\right) T_{1}^{l, t}\right)-\right.\right. \\
\left.\left.\frac{1-\hat{P}_{2}}{\left|r-r_{2}\right|}\left(\frac{1+r^{2}}{1-z_{+}} N_{2}^{l, t}+\left(r_{2}-r\right) T_{2}^{l, t}\right)\right]\right\} \frac{\alpha^{2}\left(r Q^{2}\right)}{r} .
\end{gathered}
$$

The rest of our short notation is

$$
\begin{gathered}
U^{l}=1, \quad U^{t}=\sqrt{\frac{\tau}{x \rho} \frac{1}{(x-\rho-x \rho \tau)}}, \\
W^{l}=-2 \frac{\rho \tau}{x} W, \quad W^{t}=-\frac{\rho^{2}}{x^{2}}(1+2 x \tau) W, W=[x(1+r)-1] G_{M}^{2}+\left[1+\frac{4 x \tau}{\rho r}(1+r)\right] G_{M} \widetilde{G}, \\
N_{1}^{l}=(2 \tau+r)(2-\rho) G_{M}^{2}+8 \tau\left(\frac{1-\rho}{\rho}-\frac{\tau}{r}\right) G_{M} \widetilde{G}, \\
\left.N_{2}^{l}=(2 \tau+1)(2-\rho r) G_{M}^{2}+8 \tau\left(\frac{1}{\rho r}-1-\tau\right)\right) G_{M} \widetilde{G}, \\
N_{1}^{t}=\left[1-\frac{\rho}{x}+r-\rho(r+2 \tau)\right]\left[-(2-\rho) G_{M}^{2}+2\left(1+\frac{2 \tau}{r}\right) G_{M} \widetilde{G}\right], \\
T_{1}^{l}=\left[1-\frac{\rho}{x}+\frac{1}{r}-\rho(1+2 \tau)\right]\left[-(2-\rho r) G_{M}^{2}+2(1+2 \tau) G_{M} \widetilde{G}\right], \\
T_{1}^{t}=2\left\{(r+2 \tau) G_{M}^{2}+2 \tau\left(\frac{2}{\rho}-1\right) G_{M} \widetilde{G}\right], T_{2}^{l}=-2\left[(1+2 \tau) G_{M}^{2}+2 \tau\left(\frac{2}{\rho r}-1\right) G_{M} \widetilde{G}\right], \\
\left.T_{2}^{t}=2\left[\frac{1}{r}-\rho(1+2 \tau)+1-\frac{\rho}{x}\right] G_{M}^{2}-2\left[\rho+2 \frac{\tau-1}{r}+\frac{1}{r}+1-\frac{\rho}{x}\right)\right] G_{M} \widetilde{G} .
\end{gathered}
$$

Note that the argument of the electromagnetic form factors in Eq.(67) is $-Q^{2} r$ and

$$
\widetilde{G}\left(r Q^{2}\right)=2 G_{C}\left(r Q^{2}\right)+\frac{\rho r}{6 \tau} G_{Q}\left(r Q^{2}\right)
$$

The spin-dependent Born cross sections on the right-hand side of Eq.(67) are defined by expressions (44) and (45) being multiplied by $\delta(y-\rho)$. 
Now we can write the first order correction for the spin-dependent part of the cross section by the full analogy with unpolarized case

$$
\begin{gathered}
\frac{d \sigma^{l, t}}{d Q^{2}}=\frac{d \sigma_{B}^{l, t}}{d Q^{2}}\left[1+\frac{\alpha}{2 \pi}\left(\delta(1, \rho)+(L-1) G_{0}\right)\right] \\
+\frac{\alpha}{2 \pi}(L-1) G_{1}^{l, t}+\int_{y_{\min }}^{y_{\max }}\left(H_{x}^{l, t}+H_{x r}^{l, t}\right) d y,
\end{gathered}
$$

where $G_{1}^{l, t}$ can be derived from $G_{1}$ (see Eq. (61)) by simple substitution $d \sigma \rightarrow d \sigma^{l, t}$.

\subsection{Correction to the part of the cross section caused by the tensor polarization of the} deuteron target

The radiative corrections to the polarization observables in the elastic electron-deuteron scattering caused by the tensor polarized deuteron target can be obtained using the results of the paper [56] where the model-independent radiative corrections to the deep-inelastic scattering of unpolarized electron beam off the tensor polarized deuteron target have been calculated.

To obtain the required corrections it is necessary first to get the contribution of the elastic radiative tail (the account for the radiative corrections to the elastic electron-deuteron scattering). It can be obtained using the results of the Ref. [56] where the radiative corrections to the deep-inelastic scattering of unpolarized electron beam on the tensor polarized deuteron target have been calculated. We can obtain these radiative corrections from the formula (46) of the Ref. [56] by the following substitution in the hadronic tensor

$$
B_{i}\left(q^{2}, x^{\prime}\right) \rightarrow-\frac{1}{q^{2}} \delta\left(1-x^{\prime}\right) B_{i}^{(e l)}, i=1-4,
$$

where $B_{i}\left(q^{2}, x^{\prime}\right)$ are the spin-dependent structure functions, caused by the tensor polarization of the deuteron, describing the transition $\gamma^{*} d \rightarrow X$ and the functions $B_{i}^{(e l)}$ are their elastic limit (when the final state $X$ is the deuteron). Here $q^{2}=\left(k_{1}-k_{2}-k\right)^{2}$. So, the elastic structure functions can be expressed in terms of the deuteron electromagnetic form factors as

$$
\begin{gathered}
B_{1}^{(e l)}=\bar{\eta} q^{2} G_{M}^{2}, B_{3}^{(e l)}=2 \bar{\eta}^{2} q^{2} G_{M}\left(G_{M}+2 G_{Q}\right) \\
B_{2}^{(e l)}=-2 \bar{\eta}^{2} q^{2}\left[G_{M}^{2}+\frac{4 G_{Q}}{1+\bar{\eta}}\left(G_{C}+\frac{\bar{\eta}}{3} G_{Q}+\bar{\eta} G_{M}\right)\right]
\end{gathered}
$$




$$
B_{4}^{(e l)}=-2 \bar{\eta} q^{2}(1+\bar{\eta}) G_{M}^{2}, \bar{\eta}=-q^{2} / 4 M^{2}
$$

After substitution of the elastic functions $B_{i}^{(e l)}$ into the formula (46) of the Ref. [56] we have to do an integration over $z$ variable (it shows the degree of deviation of the deep-inelastic scattering from the elastic process) using a $\delta$-function $\delta\left(1-x^{\prime}\right)=x y r \delta(z)$. Thus, the value $z=0$ corresponds to the elastic contribution (elastic electron-deuteron scattering) to the deep-inelastic process. Note that the $z$ variable used in the Ref. [56] has different meaning as compared to the $z$ variable used in this paper. And at last, to obtain the radiative corrections to the process of the elastic electron-deuteron scattering it is necessary to do integration of the elastic radiative tail contribution over the $x$ variable.

As a result we obtain the following expression for the radiatively corrected spin-dependent part of the cross section caused by the tensor polarization of the deuteron target in the elastic electron-deuteron scattering

$$
\frac{d \sigma^{Q}}{d Q^{2}}=\frac{d \sigma^{l l}}{d Q^{2}} R_{l l}+\frac{d \sigma^{t t}}{d Q^{2}}\left(R_{t t}-R_{n n}\right)+\frac{d \sigma^{l t}}{d Q^{2}} R_{l t},
$$

where the spin-dependent parts of the cross section $d \sigma^{m n}, m n=l l, l t, t t$, can be written by means of the electron structure function in the following form

$$
\begin{gathered}
\frac{d \sigma^{m n}\left(k_{1}, k_{2}, \widetilde{S}\right)}{d Q^{2}}=\int_{y_{\min }}^{y_{\max }} d y \frac{d \sigma^{m n}}{d Q^{2} d y}, \\
\frac{d \sigma^{m n}}{d Q^{2} d y}=\int_{z_{1 m}}^{1} d z_{1} \int_{z_{2 m}}^{1} \frac{d z_{2}}{z_{2}^{2}} D\left(z_{1}, L\right) \bullet \\
D\left(z_{2}, L\right) \frac{d \sigma_{\text {hard }}^{m n}\left(\tilde{k}_{1}, \tilde{k}_{2}, \widetilde{S}\right)}{d \tilde{Q}^{2} d \tilde{y}} .
\end{gathered}
$$

By full analogy with Eq.(65) one can write for the tensor partial cross-sections defined with respect to $\mathbf{L}$ and $\mathbf{T}$ directions

$$
\frac{d \sigma^{(A)}\left(k_{1}, k_{2}, S\right)}{d Q^{2}}=\int_{y_{\min }}^{y_{\max }} d y T_{A \beta}(\psi) \frac{d \sigma^{\beta}\left(k_{1}, k_{2}, \widetilde{S}\right)}{d Q^{2} d y}
$$

In this paper we define the partial tensor asymmetries in the same way as it is done for the vector ones (see Eq.(66))

$$
A_{C}^{\beta}=\frac{d \sigma^{\beta}\left(k_{1}, k_{2}, \widetilde{S}\right)}{d \sigma\left(k_{1}, k_{2}\right)}, A_{C}^{A}=\frac{d \sigma^{A}\left(k_{1}, k_{2}, S\right)}{d \sigma\left(k_{1}, k_{2}\right)},
$$


where indices $A$ and $\beta$ equal to

$$
A=L L, T T, L T ; \beta=l l, t t, l t,
$$

and the spin-dependent parts of the cross-section due to deuteron tensor polarization are determined by Eqs. (72) and (73), respectively.

The hard part of the spin-dependent cross sections which enter under the integral sign in the right-hand side of Eq.(72) can be written as

$$
\frac{d \sigma_{\text {hard }}^{m n}}{d Q^{2} d y}=\left(1+\frac{\alpha}{2 \pi} \delta(1, \rho)\right) \frac{d \sigma_{B}^{m n}}{d Q^{2} d y}+H_{x}^{m n}+H_{x r}^{m n},
$$

where we introduce the following designations

$$
\begin{gathered}
H_{x}^{m n}=\frac{\alpha}{2 \pi}\left[\frac{\left(1-r_{1}\right) \hat{P}_{1}}{1-\rho}-\frac{\left(1-r_{2}\right) \hat{P}_{2}}{r_{2}}\right] \frac{d \sigma_{B}^{m n}}{d Q^{2}}, \\
H_{x r}^{m n}=\frac{\alpha \eta}{Q^{4}}\left\{\frac{P}{1-x y} \int_{r_{-}}^{r_{+}} \frac{d r\left(1-\hat{P}_{1}\right) G^{m n}(r)}{(1-r)\left|r-r_{1}\right|}+\frac{P}{1-y(1-x)} \int_{r_{-}}^{r_{+}} \frac{d r\left(1-\hat{P}_{2}\right) \tilde{G}^{m n}(r)}{(1-r)\left|r-r_{2}\right|}\right\}- \\
\frac{\alpha \rho}{4 Q^{4}} \frac{1}{\sqrt{y^{2}+4 x y \tau}} \int_{r_{-}}^{r_{+}} d r\left[F_{0}^{m n}(r)+\xi_{1} \frac{Q^{2}}{V} F_{1}^{m n}(r)+\tau \xi_{2} \frac{Q^{4}}{V^{2}} F_{2}^{m n}(r)\right] \frac{\alpha^{2}\left(Q^{2} r\right)}{r^{2}},
\end{gathered}
$$

where the coefficients $\xi_{1,2}$ are

$$
\begin{gathered}
\xi_{1}=\frac{1}{y+4 x \tau}[1-y-2 x \tau+r(1+x y-2 x+2 x \tau)], \\
\xi_{2}=3 \xi_{1}^{2}-\frac{1}{y(y+4 x \tau)}[r(1-x y)+y-1]^{2} .
\end{gathered}
$$

The functions $G^{m n}(r), \tilde{G}^{m n}(r)$ and $F_{i}^{m n}(r), i=0,1,2$ are defined as

$$
\begin{gathered}
G^{m n}(r)=\frac{\alpha^{2}\left(Q^{2} r\right)}{r^{2}} \sum_{j=1}^{4} A_{j}^{m n} H_{j}, F_{i}^{m n}(r)=\sum_{j=1}^{4} C_{i j}^{m n} H_{j}, \\
\tilde{G}^{m n}(r)=\frac{\alpha^{2}\left(Q^{2} r\right)}{r^{2}} \sum_{j=1}^{4} B_{j}^{m n} H_{j},
\end{gathered}
$$

where the expressions for the coefficients $A_{j}^{m n}, B_{j}^{m n}$ and $C_{i j}^{m n}, m n=l l, l t, t t ; i=0,1,2 ; j=$ 1,2,3,4 are given in Appendix A. The functions $H_{j}, j=1-4$ in relations (76) depend on the shifted momentum transverse squared, i.e., $H_{j}=H_{j}\left(r Q^{2}\right)$ and

$$
\begin{gathered}
H_{1}\left(Q^{2}\right)=G_{M}^{2}, \quad H_{4}\left(Q^{2}\right)=-2(1+\eta) G_{M}^{2} \\
H_{2}\left(Q^{2}\right)=-2 \eta\left[G_{M}^{2}+\frac{4}{1+\eta} G_{Q}\left(G_{C}+\frac{\eta}{3} G_{Q}+\eta G_{M}\right)\right]
\end{gathered}
$$




$$
H_{3}\left(Q^{2}\right)=2 \eta G_{M}\left(G_{M}+2 G_{Q}\right) .
$$

Now we can extract from the master formula (72) the first order correction for the spindependent parts of the cross section, caused by the tensor polarization of the deuteron target

$$
\begin{gathered}
\frac{d \sigma^{m n}}{d Q^{2}}=\left\{1+\frac{\alpha}{2 \pi}\left[\delta(1, \rho)+(L-1) G_{0}\right]\right\} \frac{d \sigma_{B}^{m n}}{d Q^{2}}+ \\
\frac{\alpha}{2 \pi}(L-1) G_{1}^{m n}+\int_{y_{\min }}^{y_{\max }}\left(H_{x}^{m n}+H_{x r}^{m n}\right) d y,
\end{gathered}
$$

here $m n=l l, l t, t t$ and the rest of designations are

$$
\begin{gathered}
G_{1}^{m n}=I_{1}^{m n}(z)+I_{2}^{m n}(\tilde{z}), \\
I_{1}^{m n}(z)=-\int_{1-z}^{1} d r_{1} \frac{\left(1+r_{1}^{2}\right)\left(1-\hat{P}_{1}\right)}{1-r_{1}} \frac{d \sigma_{B}^{m n}}{d Q^{2}}, \\
I_{2}^{m n}(\tilde{z})=-\int_{\frac{1}{1-\tilde{z}}}^{1} \frac{d r_{2}}{r_{2}^{3}} \frac{\left(1+r_{2}^{2}\right)\left(1-\hat{P}_{2}\right)}{1-r_{2}} \frac{d \sigma_{B}^{m n}}{d Q^{2}} .
\end{gathered}
$$

\section{NUMERICAL ESTIMATIONS}

The recent measurements in polarized electron-deuteron scattering are the following: i)measurement of analyzing power $T_{20}$ in region $0.126 \mathrm{GeV}^{2}<Q^{2}<0.397 \mathrm{GeV}^{2}$ [12]; ii) measurement of recoil polarizations $t_{20}, t_{21}, t_{22}$ at $0.66 \mathrm{GeV}^{2}<Q^{2}<1.7 \mathrm{GeV}^{2}$ [16]; iii) measurement of analyzing powers $T_{20}, T_{21}, T_{22}$ at $0.326 \mathrm{GeV}^{2}<Q^{2}<0.838 \mathrm{GeV}^{2}$.

For the deuteron form factors we use the results of the paper [57] where the world data for elastic electron-deuteron scattering was used to parameterize, in three different ways, the three electromagnetic form factors of the deuteron in the four-momentum transfer range $0-7 \mathrm{fm}^{-1}$. The accuracy in the determination of these form factors is limited by the assumption of a one-photon exchange mechanism and precise calculation of the radiative corrections. In the intermediate to high $Q$-range, other corrections such as the double scattering contribution to two-photon exchange [30] should be considered, but they are at present neither accurately calculated nor experimentally determined.

For numerical calculations we use two different parameterizations labeled as I and II. In the parametrization I, each form factor is given by polynomial in $Q^{2}$ variable. With 18 free parameters, a fit was obtained with $\chi^{2} / N_{\text {d.f. }}=1.5$. The parametrization II has been 
proposed in Ref. [58]. Each form factor is proportional to the square of a dipole nucleon form factor and to a linear combination of reduced helicity transition amplitudes. In addition, an asymptotic behavior dictated by quark counting rules and helicity rules valid in perturbative QCD were incorporated in the fitting procedure. With 12 free parameters, a fit to the data set was obtained with $\chi^{2} / N_{\text {d.f. }}=1.8$, whereas the original values of the parameters in Ref. [58] yield $\chi^{2} / N_{\text {d.f. }}=7.5$. The parametrization II, in contrast with the two other ones presented in this paper, can be extrapolated well above $7 \mathrm{fm}^{-1}$, albeit with some theoretical prejudice.

To demonstrate effect of radiative corrections in the considered polarized phenomena we give the $Q^{2}$-dependence of quantities $\delta A^{I}$ and $\delta A^{I J}$ defined as

$$
\delta A^{I}=A_{C}^{I}-A_{B}^{I}, \delta A^{I J}=A_{C}^{I J}-A_{B}^{I J},
$$

where $A_{C}^{I}, A_{C}^{I J}$ designate the values of the corresponding asymmetries with accounting of the radiative corrections (see Eqs. (66) and (74)) and $A_{B}^{I}, A_{B}^{I J}$ are their Born values.

During calculation we took $V=2\left(k_{1} p_{1}\right)=10 \mathrm{GeV}^{2}$ and $0.1 \mathrm{GeV}^{2} \leq Q^{2} \leq 2 \mathrm{GeV}^{2}$ and used parametrization I and II of the deuteron form factors given in Ref.[57]. It turns out that the difference between the asymmetries calculated with these two parametrizations are very small and further we use the parametrization I.
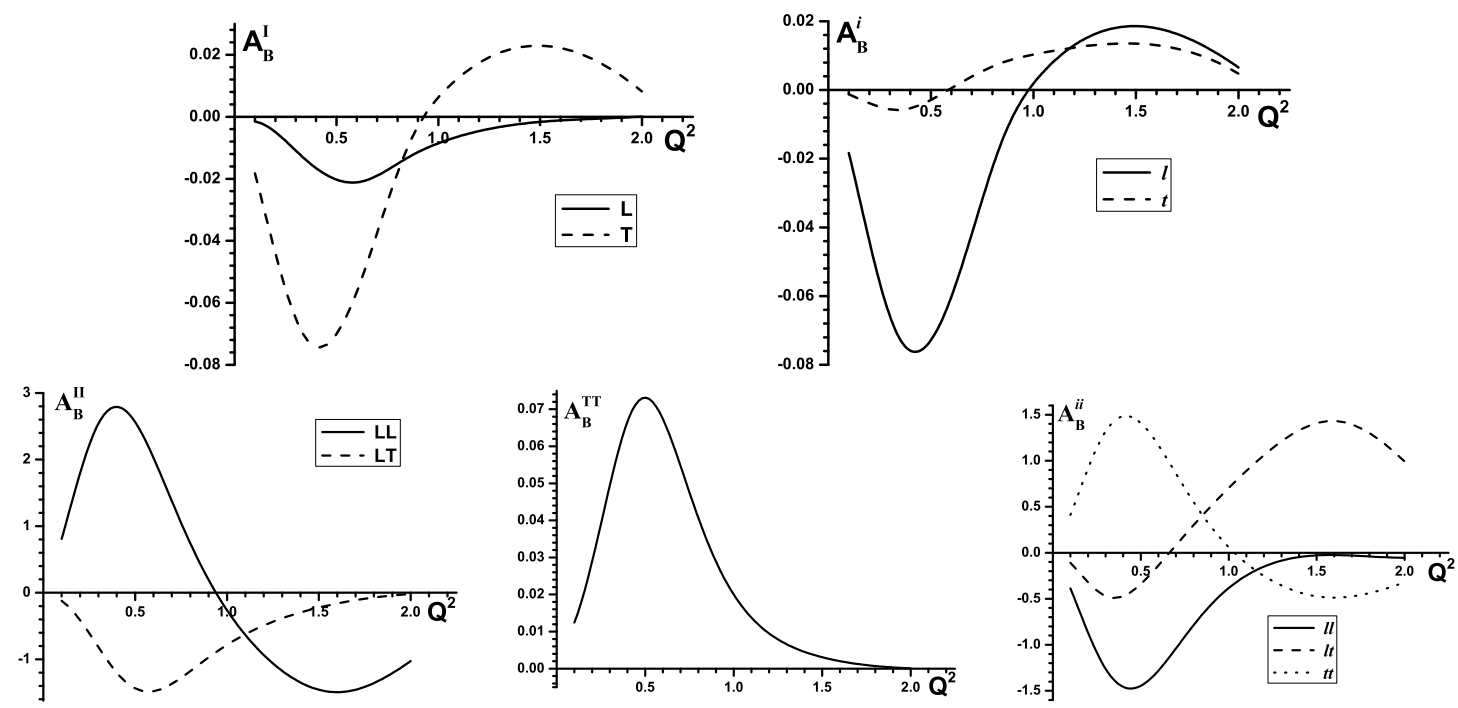

Fig.1.

The Born values of vector and tensor asymmetries calculated by means equations (25), (26) and (34). Note that the quantity $A_{B}^{T T}$ is small as compared with $A_{B}^{t t} . Q^{2}$ is given in $\mathrm{GeV}^{2}$. 
In Fig.1 the Born values of vector and tensor asymmetries are shown. As one can see the absolute values of the vector asymmetries are small as compared with the tensor ones. Besides, effect of the polarization direction choice is seen very clear. The most expressive one is that $A_{B}^{T T}$ is near zero at all considered values of $Q^{2}$, whereas the corresponding quantity $A_{B}^{t t}$ is large enough (of the order of 1 ).

In Fig. 2 we demonstrate the influence of radiative corrections on the single-spin tensor asymmetries. The corresponding effect depends strongly on parameter $\Delta_{t h}$ which defines the rules for event selection. If one takes $\Delta_{t h}=0$, the radiation of hard photons is forbidden, and effect vanishes as it follows from master formulas (55), (64) and (72) as well as from Eqs.(65)and (73).
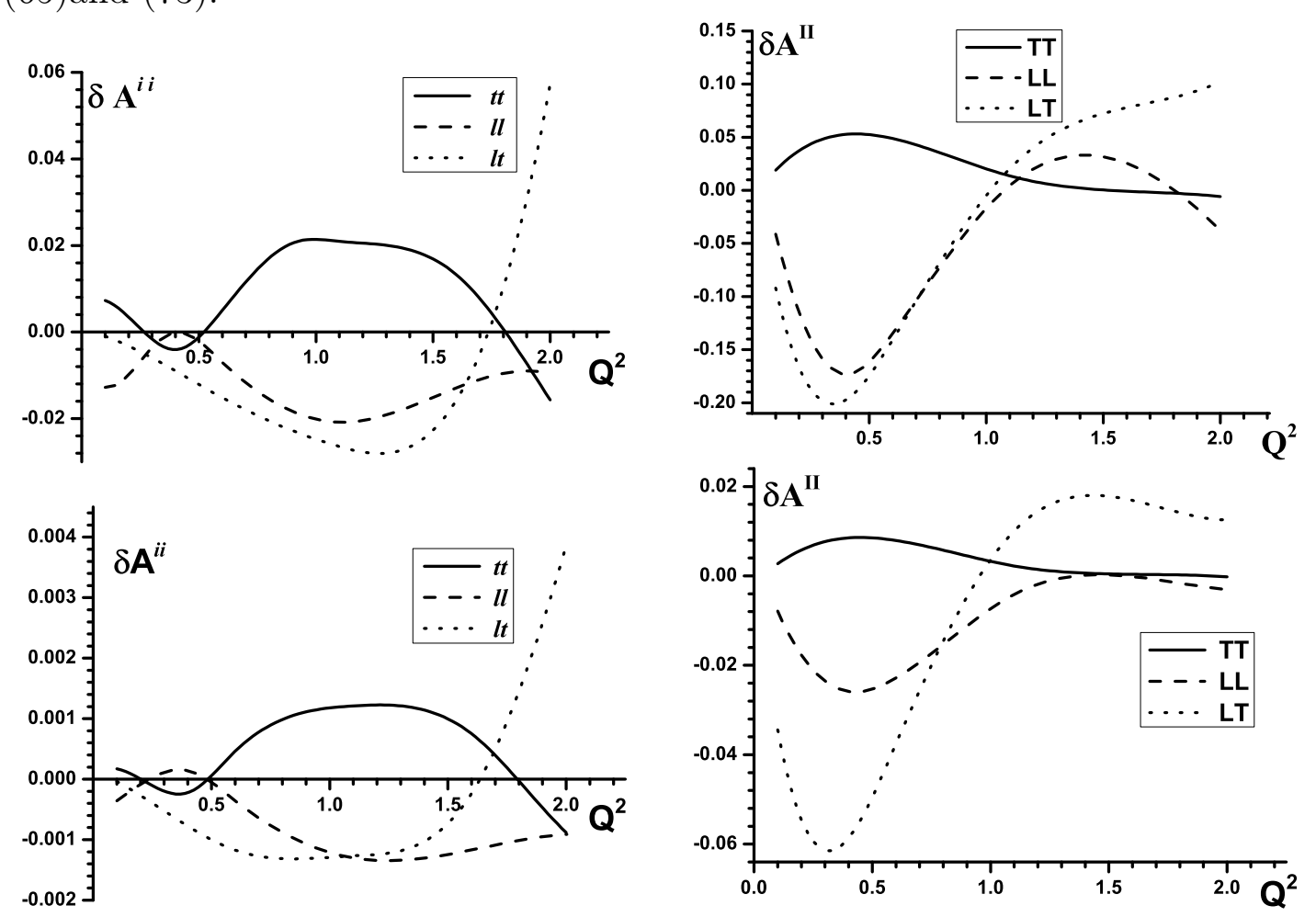

Fig.2.

Effect of radiative corrections for the single-spin tensor asymmetries defined with respect to the stable (left column) and unstable (right column) directions at radiation of the collinear photons and $e^{+} e^{-}$- pairs by the initial and final electrons. The curves in the upper row are calculated at $\Delta_{t h}=0.26$ and in the low one at $\Delta_{t h}=0.0526$. In Born approximation all quantities $\delta \mathbf{A}^{\mathbf{i i}}$ and $\delta \mathbf{A}^{\mathbf{I I}}$ equal to zero. $Q^{2}$ is given in $\mathrm{GeV}^{2}$. 

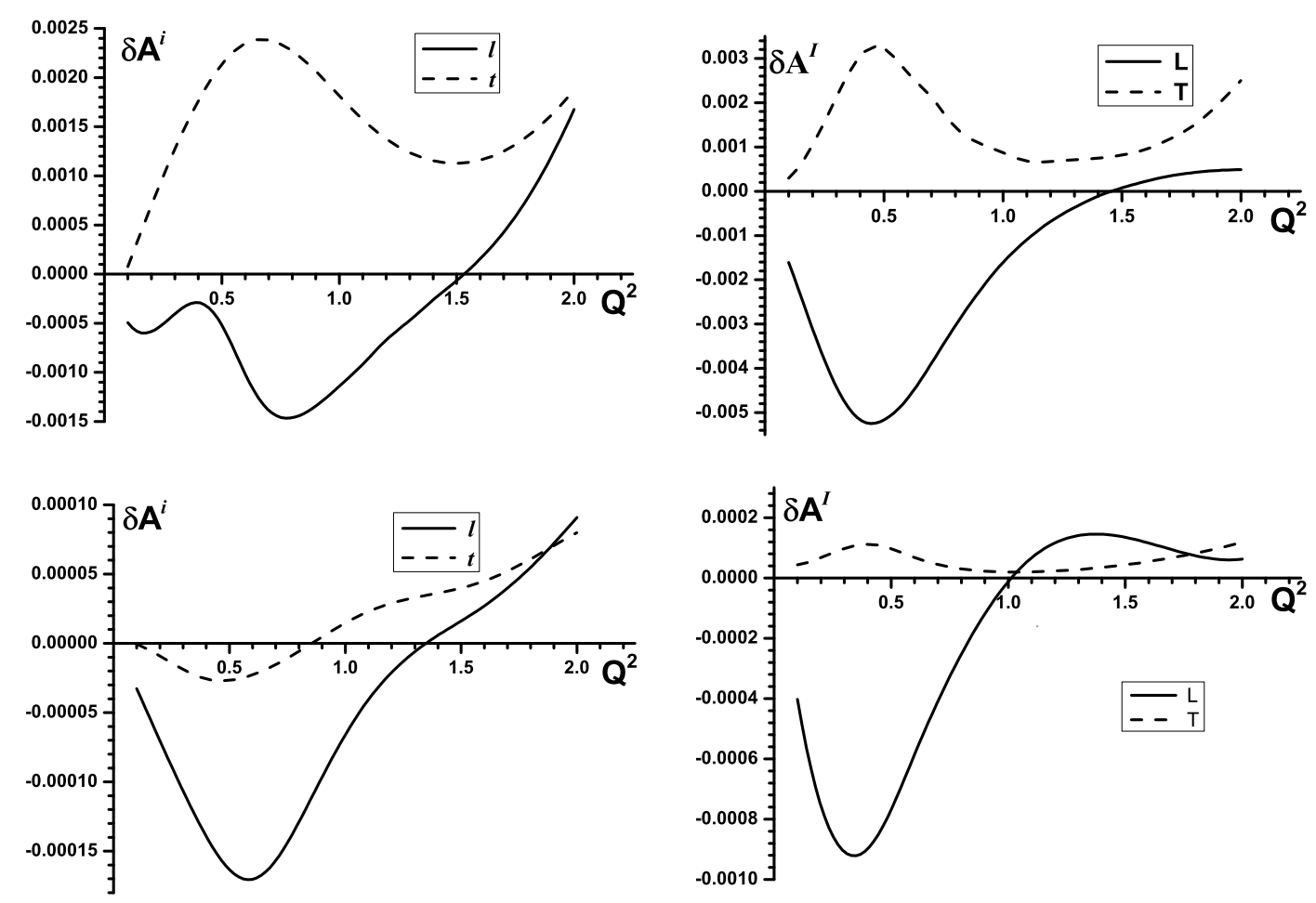

Fig.3.

The same as in Fig.2 but for the case of the double-spin vector asymmetries.

In this case the radiative corrections are determined by the soft photon emission and virtual loops and are factorized in both unpolarized cross-section and polarization dependent ones. If the hard photon emission is allowed but the pion production threshold does not exceeded $\Delta_{t h} \leq 0.0526$ (see Eqs.(57) and (58)) the absolute value of the effect is smaller than $2.5 \%$. With increase of the allowed photon energy the tensor asymmetries can reach the values of the order of $15-20 \%$.

As concern the double-spin vector asymmetries, they are very small even at the Born level, and up to now there is not any attempt to measure them. We calculate them to give the complete picture of accounting for model-independent radiative corrections in electrondeuteron scattering.

In Sec.2 we stressed that measurement of the polarization asymmetries in process (1), in principle, can be used to determine the deuteron magnetic form factor $G_{M}$. The reason is that the quantities $A_{B}^{L}$ (see Eq.(26)) and $A_{B}^{L T}$ (see Eq. (34)) are proportional to $G_{M}^{2}$ if the deuteron polarization states are determined with respect to directions defined by Eq.(21), when the longitudinal direction is chosen along the 3-momentum transferred. If 
these states are defined by Eq.(39), when the longitudinal direction is chosen along the initial electron 3-momentum, such simple form of mentioned asymmetries is violated, and analysis of polarization data has became more complicate. Such situation is conserved if the corrections due to soft photon emission and virtual loop are taken into account. But inclusion of radiative events with hard photon emission (process (52)) inevitably changes it because even radiation of collinear photons alters the direction of the 3-momentum transferred, and the rotation of polarization states necessary occurs.

To take into account the radiative corrections in this case, by the electron structure function method, one needs, according to the spirit of this method, to use the set of polarization states that are stable under collinear photon radiation by both, initial and scattered, electrons. The corresponding spin-dependent parts of the hard cross-sections in master formulas (64) and (72) include different combinations of all deuteron form factors. It means, for example, that small partial cross-section $d \sigma^{T T} / d Q^{2}$ which expressed through the large ones $d \sigma^{t t} / d Q^{2}, d \sigma^{l l} / d Q^{2}$ and $d \sigma^{l t} / d Q^{2}$ can be significantly changed if undetected additional particles accompany the process (1). At least for $\Delta_{t h}=0.26$ the radiative corrections almost double the tensor asymmetry $A^{T T}$ as compared with the Born one. With decrease of $\Delta_{t r}$ this effect is diminished. Note that similar effect is absent if one uses the hadronic variables to describe radiative corrections (see Eqs. (64) and (69) in Ref. [35]). The reason is that in this case the recoil deuteron momentum is measured independently on undetected particles in leptonic part of interaction.

In present paper we give the consistent calculation of electromagnetic model-independent radiative corrections for polarization observables in the process of elastic electron-deuteron scattering. Our approach based on the electron structure function method and covariant description of the polarization states, provided the event selection carried out by restriction on the lost invariant mass. The only additional parameter, in this case, that required to be determined in measurements is $\Delta_{t h}$. Usually in real experiments the rules for event selections can include different cuts caused by the measurement method and the detector geometry. Every cut leaves the trace on the level of radiative corrections if undetected particles are allowed. Thus, in every independent experiment radiative corrections are different since the cut procedure is distinguished in the general, and only Monte Carlo event generator can take into account all the restrictions exactly. Our semi-analytical result can be incorporated in such generator to check its work for considered event selection. 


\section{APPENDIX A}

In this Appendix we present the formulae for the coefficients $A_{i}^{m n}, B_{i}^{m n}$ and $C_{i j}^{m n},(m n=$ $l l, l t, t t ; i=0,1,2 ; j=1,2,3,4)$ that determining the partial cross- sections in the case of tensor polarized target (see formula $(75)$ ).

\section{Component $l l$}

The coefficients, determining the contribution proportional to the components $R_{l l}$ of the tensor that describe the tensor polarization of the deuteron target, can be written as:

$$
\begin{aligned}
& A_{1}^{l l}=-x y\left(1+r^{2}\right) Z, \quad A_{2}^{l l}=\frac{Z Z_{1}}{x y r}, \\
& A_{3}^{l l}=\frac{1}{x y}\left\{(a+\bar{r})\left[2 Z_{1}+r \Delta_{1}\left(2 a+r-\Delta_{1}\right)\right]-\right. \\
& \left.\Delta_{1}\left[r^{2}\left(r-\Delta_{1}\right)+2\left(b+\Delta_{1}\right)+r(a+b)\left(a+r-\Delta_{1}\right)\right]\right\} \text {, } \\
& A_{4}^{l l}=r\left\{(b-a)\left(1+r^{2}\right)+\Delta_{1}[1+r(2 a-b)]\right\}, \\
& B_{1}^{l l}=x y r\left(1+r^{2}\right)[x y r(1+6 \tau)-2 \tau(1-3 a r)], \\
& B_{2}^{l l}=-\frac{1}{x y}[x y r(1+6 \tau)-2 \tau(1-3 a r)] \text {. } \\
& {\left[b\left(1+r^{2}\right)-\Delta_{2}(\bar{r}-2 a)\right]} \\
& B_{3}^{l l}=-\frac{1}{x y}\left\{2 Z_{2}\left[1+(2 a-b) r+\Delta_{2}\right]+\right. \\
& \left.3 a \Delta_{2}\left[(b-a) r-1-\Delta_{2}\right]\right\} \text {, } \\
& B_{4}^{l l}=r\left[(a-b)\left(1+r^{2}\right)+\Delta_{2}(a+\bar{r})\right], \\
& C_{01}^{l l}=\frac{2}{\tau}\left\{\left(\bar{r}-\Delta_{1}\right)^{2}+a\left[3 a\left(1+r^{2}\right)-2\left(b+\Delta_{1}\right)\right]\right\}, \\
& C_{02}^{l l}=\frac{2}{x y r}\left\{(7-3 y)(x y r)^{2}+3 a(5-y+r) x y r+\right. \\
& \left.3 a^{2}\left(3+r^{2}\right)-\operatorname{ar}\left[5+3(a+b)^{2}\right]\right\}, \\
& C_{03}^{l l}=-x y[r(6 a-16+9 y)+6 \tau(y-3-r)], \\
& C_{04}^{l l}=x y r[1+3(b-a)], C_{11}^{l l}=12 x y(r+2 \tau),
\end{aligned}
$$




$$
\begin{gathered}
C_{12}^{l l}=6 \frac{\tau}{r}[4(r+\tau)-y r], \quad C_{13}^{l l}=6 \tau(2-y), \\
C_{21}^{l l}=6, C_{22}^{l l}=\frac{6 \tau}{x y r}, \quad C_{14}^{l l}=C_{23}^{l l}=C_{24}^{l l}=0 .
\end{gathered}
$$

\section{Component lt}

The coefficients, determining the contribution proportional to the components $R_{l t}$ of the tensor that describe the tensor polarization of the deuteron target, can be written as:

$$
\begin{aligned}
& A_{1}^{l t}=2 a(2 \tau+r)\left(1+r^{2}\right)\left(2 b+\Delta_{1}\right) \frac{Q^{2}}{M d}, \\
& A_{2}^{l t}=-2(2 \tau+r)\left(2 b+\Delta_{1}\right) Z_{1} \frac{\tau}{r} \frac{V}{M d}, \\
& A_{3}^{l t}=-\tau \frac{V}{M d}\left\{2 Z_{1}(3 b-a-r)+\Delta_{1}\left[4 r\left(1+b^{2}+3 a b\right)\right.\right. \\
& \left.\left.-2 a\left(1+r^{2}\right)+x y r(a r-3+5 b r)\right]\right\}, \\
& A_{4}^{l t}=\operatorname{ar} \frac{V}{M d}\left\{2 b\left(1+r^{2}\right)+\Delta_{1}[1-r(3 b-a)]\right\}, \\
& B_{1}^{l t}=2 a r(1+2 \tau)\left(1+r^{2}\right)\left(\Delta_{2}-2 b r\right) \frac{Q^{2}}{M d}, \\
& B_{2}^{l t}=-2 \tau(1+2 \tau)\left(\Delta_{2}-2 b r\right) Z_{2} \frac{V}{M d}, \\
& B_{3}^{l t}=\tau \frac{V}{M d}\left\{2 Z_{2}[(3 b-a) r-1]+\Delta_{2}[(1+a r)(a-6 b)\right. \\
& \left.\left.-(a+3 b)\left(r^{2}+\Delta_{2}\right)+r\left(b^{2}-1\right)+b+\Delta_{2}(3 r-2 b)\right]\right\}, \\
& B_{4}^{l t}=\operatorname{ar} \frac{V}{M d}\left[-2 b\left(1+r^{2}\right)+\Delta_{2}(\bar{r}-2 b)\right], \\
& C_{01}^{l t}=\frac{4 Q^{2}}{M d}\left[a\left(1+r^{2}\right)(y+2 a)-2 b \bar{r}-\Delta_{1}(x y r+2 a-2 b)\right], \\
& C_{02}^{l t}=\frac{-2 V}{M d x y r}\{2 a x y r[y \bar{r}+(3 b+a)(1+r)-y-8 a] \\
& -(x y r)^{2}[2 a+(2-y)(y+4 a)]+2 a[2 a(b-a+r)+ \\
& \left.\left.(y+2 a)\left(r-a\left(1+r^{2}\right)+r(a+b)^{2}\right)\right]\right\}, \\
& C_{03}^{l t}=-\frac{Q^{2}}{M d}\left\{4 \tau\left[2 b \bar{r}-y^{2}+4\left(b^{2}-a\right)\right]-\right. \\
& r[3 y(2-y)+8 a(1+a+2 b)]\},
\end{aligned}
$$




$$
\begin{gathered}
C_{04}^{l t}=x y r \frac{V}{M d}\left[1+4 a b-(a-b)^{2}\right], \\
C_{11}^{l t}=\frac{4 Q^{2}}{M d}[2 \tau(2 y+4 a-1)+r(y+4 a-2 \tau)], \\
C_{12}^{l t}=\frac{4 \tau V}{M d r}[(a-b)(4 \tau-y r)+2 \tau(1-r)+2 y r(1+4 x \tau)], \\
C_{13}^{l t}=4 \tau(2-y)(y+2 a) \frac{V}{M d}, C_{14}^{l t}=C_{23}^{l t}=C_{24}^{l t}=0, \\
C_{21}^{l t}=4(y+2 a) \frac{V}{M d}, C_{22}^{l t}=4 \tau \frac{y+2 a}{x y r} \frac{V}{M d} .
\end{gathered}
$$

\section{Component tt}

The coefficients, determining the contribution proportional to the components $R_{t t}$ of the tensor that describe the tensor polarization of the deuteron target, can be written as:

$$
\begin{gathered}
A_{1}^{t t}=-\frac{a}{b}\left(1+r^{2}\right)\left[b^{2}+\left(b+\Delta_{1}\right)^{2}\right], \\
A_{2}^{t t}=\frac{\tau}{b} \frac{Z_{1}}{x y r}\left[2 b^{2}+\Delta_{1}\left(2 b+\Delta_{1}\right)\right], A_{4}^{t t}=-a r^{2} \Delta_{1}, \\
A_{3}^{t t}=\frac{\tau}{b}\left\{\left(\Delta_{1}-2 b\right)\left[b\left(1+r^{2}\right)+(1-r+r y) \Delta_{1}\right]+\right. \\
\left.b \Delta_{1}\left[1+r\left(b-a+\Delta_{1}\right)\right]\right\} \\
B_{1}^{t t}=\frac{a}{b}\left(1+r^{2}\right)\left[2 b^{2} r^{2}-2 b r \Delta_{2}+\Delta_{2}^{2}\right], \\
B_{2}^{t t}=-\frac{\tau}{b} \frac{Z_{2}}{x y r}\left[2 b^{2} r^{2}-2 b r \Delta_{2}+\Delta_{2}^{2}\right], B_{4}^{t t}=-a r \Delta_{2}, \\
B_{3}^{t t}=\frac{\tau}{b}\left\{\left(2 b r-\Delta_{2}\right) Z_{2}+b \Delta_{2}\left[r(b-a+r)-\Delta_{2}\right]\right\} \\
C_{01}^{t t}=\frac{x y}{b}\left[\left(1+r^{2}\right)\left(y^{2}+4 a-2 a b\right)+\left(2 b+\Delta_{1}\right)^{2}+\Delta_{1}^{2}\right] \\
C_{02}^{t t}=-\frac{1}{b x y r}\left\{-2(x y r)^{2}[a+(1+a)(2-y)]+x y r[(3-2 y\right. \\
\left.\left.+a^{2}+b^{2}\right)(\bar{r}-2 a)+4\left(a b+b-a^{2}\right)+4 r\left(a-b^{2}\right)\right]- \\
-2 a\left[(r-a)^{2}+b^{2}\right]+(1+2 a-2 b+ \\
\left.\left.a^{2}+b^{2}\right)\left[r-a\left(1+r^{2}\right)+(a+b)^{2} r\right]\right\} \\
C_{03}^{t t}=-\frac{1}{b}\left\{3 b-a-\left(a^{2}+b^{2}\right)(2+a+b)+r\left[y^{2}+2 y(2 b-a)+\right.\right.
\end{gathered}
$$




$$
\begin{gathered}
2 a(3-a)]+x y r[y(1+y+3 a)-4(1+a)-2 a b]\}, \\
C_{04}^{t t}=x y r(y+2 a), \\
C_{11}^{t t}=-\frac{4}{b}[y(b-a+r)+2 a(r-a)-x y r(1+a)], \\
C_{12}^{t t}=\frac{1}{b x y r}\left\{r \left[1+7 a(1+a)-b(1+b)+(a+b)\left(a^{2}+\right.\right.\right. \\
\left.\left.\left.b^{2}\right)\right]+4 \tau\left[(a-b)(1-r)+a^{2}+b^{2}-r\right]\right\}, \\
C_{13}^{t t}=\frac{1}{b x y}(2-y)\left[y^{2}+2 a(2-b)\right], C_{14}^{t t}=C_{23}^{t t}=C_{24}^{t t} 0, \\
C_{21}^{t t}=\frac{1}{a b}\left[y^{2}+2 a(2-b)\right], C_{22}^{t t}=\frac{1}{b r x^{2} y^{2}}\left[y^{2}+2 a(2-b)\right],
\end{gathered}
$$

here we use the following notation

$$
\begin{gathered}
\Delta_{1}=(1-x y) r-a-b, \Delta_{2}=(1-y+x y) r-1, \\
Z_{1}=b\left(1+r^{2}\right)+\Delta_{1}(1-r+y r), Z_{2}=b\left(1+r^{2}\right)+\Delta_{2}(1-y-r), \\
Z=x y(2 \tau+r)^{2}-2 \tau\left(b+\Delta_{1}\right), \\
a=x y \tau, b=1-y-a, \bar{r}=a-b+r .
\end{gathered}
$$

\section{APPENDIX B}

Now we give some formulae describing the polarization state of the deuteron target for different cases. For the case of arbitrary polarization of the target it is described by the general spin-density matrix (in general case it is defined by 8 parameters) which in the coordinate representation has the form

$$
\begin{gathered}
\rho_{\mu \nu}=-\frac{1}{3}\left(g_{\mu \nu}-\frac{p_{\mu} p_{\nu}}{M^{2}}\right)+\frac{i}{2 M} \varepsilon_{\mu \nu \lambda \rho} s_{\lambda} p_{\rho}+Q_{\mu \nu}, \\
Q_{\mu \nu}=Q_{\nu \mu}, \quad Q_{\mu \mu}=0, \quad p_{\mu} Q_{\mu \nu}=0,
\end{gathered}
$$

where $p_{\mu}$ is the deuteron 4-momentum, $s_{\mu}$ and $Q_{\mu \nu}$ are the deuteron polarization 4-vector and the deuteron quadrupole-polarization tensor.

In the deuteron rest frame the above formula is written as

$$
\rho_{i j}=\frac{1}{3} \delta_{i j}-\frac{i}{2} \varepsilon_{i j k} s_{k}+Q_{i j}, i j=x, y, z .
$$


This spin-density matrix can be written in the helicity representation using the following relation

$$
\rho_{\lambda \lambda^{\prime}}=\rho_{i j} e_{i}^{(\lambda) *} e_{j}^{\left(\lambda^{\prime}\right)}, \rho_{\lambda \lambda^{\prime}}=\left(\rho_{\lambda^{\prime} \lambda}\right)^{*}, \lambda, \lambda^{\prime}=+,-, 0,
$$

where $e_{i}^{(\lambda)}$ are the deuteron spin functions which have the deuteron spin projection $\lambda$ on to the quantization axis ( $\mathrm{z}$ axis). They are

$$
e^{( \pm)}=\mp \frac{1}{\sqrt{2}}(1, \pm i, 0), e^{(0)}=(0,0,1)
$$

The elements of the spin-density matrix in the helicity representation are related to the ones in the coordinate representation by such a way

$$
\begin{gathered}
\rho_{++}=\frac{1}{3}+\frac{1}{2} s_{z}-\frac{1}{2} Q_{z z}, \rho_{--}=\frac{1}{3}-\frac{1}{2} s_{z}-\frac{1}{2} Q_{z z}, \\
\rho_{00}=\frac{1}{3}+Q_{z z}, \rho_{+-}=-\frac{1}{2}\left(Q_{x x}-Q_{y y}\right)+i Q_{x y} \\
\rho_{+0}=\frac{1}{2 \sqrt{2}}\left(s_{x}-i s_{y}\right)-\frac{1}{\sqrt{2}}\left(Q_{x z}-i Q_{y z}\right), \\
\rho_{-0}=\frac{1}{2 \sqrt{2}}\left(s_{x}+i s_{y}\right)-\frac{1}{\sqrt{2}}\left(Q_{x z}+i Q_{y z}\right) .
\end{gathered}
$$

To obtain this relations we used $Q_{x x}+Q_{y y}+Q_{z z}=0$.

The polarized deuteron target which is described by the population numbers $n_{+}, n_{-}$and $n_{0}$ is often used in the spin experiments (see, for example, Ref. [59]). Here $n_{+}, n_{-}$and $n_{0}$ are the fractions of the atoms with the nuclear spin projection on to the quantization axis $m=+1, \quad m=-1$ and $m=0$, respectively. If the spin-density matrix is normalized to 1 , i.e., $\operatorname{Tr} \rho=1$, then we have $n_{+}+n_{-}+n_{0}=1$. Thus, the polarization state of the deuteron target is defined in this case by two parameters: the so-called $\mathrm{V}$ (vector) and $\mathrm{T}$ (tensor) polarizations

$$
V=n_{+}-n_{-}, T=1-3 n_{0}
$$

Using the definitions for the quantities $n_{ \pm, 0}$

$$
n_{ \pm}=\rho_{i j} e_{i}^{( \pm) *} e_{j}^{( \pm)}, n_{0}=\rho_{i j} e_{i}^{(0) *} e_{j}^{(0)}
$$

we have the following relation between $V$ and $T$ parameters and parameters of the spindensity matrix in the coordinate representation (in the case when the quantization axis is directed along the $\mathrm{z}$ axis)

$$
n_{0}=\frac{1}{3}+Q_{z z}, n_{ \pm}=\frac{1}{3} \pm \frac{1}{2} s_{z}-\frac{1}{2} Q_{z z}
$$


or

$$
T=-3 Q_{z z}, V=s_{z}
$$

Now let us relate the parameters of the density matrix for the massive particle of spin-one (deuteron) for two representations: the coordinate (see Eq. (B.1)) and spherical tensors.

According to Madison Convention [60]the density matrix of a spin-one particle is given by the expression

$$
\rho=\frac{1}{3} \sum_{k q} t_{k q}^{*} \tau_{k q},
$$

where $t_{k q}$ are the polarization parameters of the deuteron density matrix and $\tau_{k q}$ are the spherical tensors. The latter ones are expressed as

$$
\begin{gathered}
\tau_{00}=1, \tau_{10}=\sqrt{\frac{3}{2}} S_{z}, \tau_{1 \pm 1}=\mp \frac{\sqrt{3}}{2}\left(S_{x} \pm i S_{y}\right), \\
\tau_{20}=\frac{3}{\sqrt{2}}\left(S_{z}^{2}-\frac{2}{3}\right), \tau_{2 \pm 2}=\frac{\sqrt{3}}{2}\left(S_{x} \pm i S_{y}\right)^{2} \\
\tau_{2 \pm 1}=\mp \frac{\sqrt{3}}{2}\left[\left(S_{x} \pm i S_{y}\right) S_{z}+S_{z}\left(S_{x} \pm i S_{y}\right)\right] \\
S_{x}=\frac{1}{\sqrt{2}}\left(\begin{array}{lll}
0 & 1 & 0 \\
1 & 0 & 1 \\
0 & 1 & 0
\end{array}\right), S_{y}=\frac{1}{\sqrt{2}}\left(\begin{array}{ccc}
0 & -i & 0 \\
i & 0 & -i \\
0 & i & 0
\end{array}\right), S_{z}=\left(\begin{array}{ccc}
1 & 0 & 0 \\
0 & 0 & 0 \\
0 & 0 & -1
\end{array}\right) .
\end{gathered}
$$

From Eq. (B.11) and hermiticity of the spin operator we immediately get

$$
\tau_{k q}^{+}=(-1)^{q} \tau_{k-q}
$$

and the hermiticity condition for the density matrix yields for $\tau_{k q}$

$$
t_{k q}^{*}=(-1)^{q} t_{k-q}
$$

From this equation one can see that

$$
\begin{gathered}
t_{10}^{*}=t_{10}, t_{11}^{*}=-t_{1-1}, t_{20}^{*}=t_{20}, \\
t_{22}^{*}=t_{2-2}, t_{21}^{*}=-t_{2-1},
\end{gathered}
$$

i.e., the parameters $t_{10}$ and $t_{20}$ are real ones, and the parameters $t_{11}, t_{21}$ and $t_{22}$ are complex ones. So, in total there are 8 independent real parameters as it must to be for the spin-one massive particle. 
After that we come to explicit expression of the deuteron density matrix

$$
\rho=\frac{1}{3}\left(\begin{array}{ccc}
1+\sqrt{\frac{3}{2}} t_{10}+\frac{1}{\sqrt{2}} t_{20} & \sqrt{\frac{3}{2}}\left(t_{1-1}+t_{2-1}\right) & \sqrt{3} t_{2-2} \\
-\sqrt{\frac{3}{2}}\left(t_{11}+t_{21}\right) & 1-\sqrt{2} t_{20} & \sqrt{\frac{3}{2}}\left(t_{1-1}-t_{2-1}\right) \\
\sqrt{3} t_{22} & -\sqrt{\frac{3}{2}}\left(t_{11}-t_{21}\right) & 1-\sqrt{\frac{3}{2}} t_{10}+\frac{1}{\sqrt{2}} t_{20}
\end{array}\right) .
$$

The density matrix is normalized to 1 , i.e., $\operatorname{Tr} \rho=1$. Using the expression for the density matrix in the helicity representation, Eq. (B.5) we get the following relations between the parameters of the density matrix in the coordinate representation and spherical tensor one

$$
\begin{gathered}
t_{10}=\sqrt{\frac{3}{2}} s_{z}, \operatorname{Ret}_{11}=-\operatorname{Ret}_{1-1}=-\frac{\sqrt{3}}{2} s_{x}, \operatorname{Imt}_{11}=\operatorname{Imt}_{1-1}=-\frac{\sqrt{3}}{2} s_{y}, \\
t_{20}=-\frac{3}{\sqrt{2}} Q_{z z}, \operatorname{Ret}_{21}=-\operatorname{Ret}_{2-1}=\sqrt{3} Q_{x z}, \operatorname{Imt}_{21}=\operatorname{Imt}_{2-1}=\sqrt{3} Q_{y z}, \\
\operatorname{Ret}_{22}=\operatorname{Ret}_{2-2}=-\frac{\sqrt{3}}{2}\left(Q_{x x}-Q_{y y}\right), \operatorname{Imt}_{22}=-\operatorname{Imt}_{2-2}=-\sqrt{3} Q_{x y} .
\end{gathered}
$$

1. M. Garcon, Preprint DAPNIA/SPHN-99-77, Saclay, 1999.

2. M. Garcon and J. W. Van Orden, arXiv: nucl-th/0102049.

3. Ingo Sick, arXiv: nucl-ex/0208009.

4. R. Gilman, F. Gross, J. Phys. G: Nucl. Part. Phys. 28, R37 (2002).

5. M. Kohl, Nucl. Phys. A805, 361c (2008).

6. S. Platchkov et al., Nucl. Phys. A510, 740 (1990); E. E. W. Bruins et al., Phys. Rev. Lett. 75, 21 (1995).

7. HERMES Collaboration: Ackerstaff et al., Phys. Lett. B404, 383 (1997).

8. M. Ferro-Luzzi et al., Nucl. Phys. A631, 190c (1998).

9. V. F. Dmitriev et al., Phys. Lett. B157, 143 (1985).

10. R. Gilman et al., Phys. rev. Lett. 65, 1733 (1990).

11. M. Ferro-Luzzi et al., Phys. Rev. Lett. 77, 2630 (1996).

12. M. Bouwhuis et al., Phys. Rev. Lett. 82, 3755 (1999).

13. N. Nikolenko et al., Phys. Rev. Lett. 90, 072501 (2003); Nucl. Phys. A721, 409c (2003).

14. M. E. Schulze et al., Phys. Rev. Lett. 52, 597 (1984).

15. M. Garcon et al., Phys. Rev. C49, 2516 (1994); 
16. D. Abbott et al., Phys. Rev. Lett. 84, 5053 (2000).

17. Egle Tomasi-Gustafsson, M. P. Rekalo, arXiv:nucl-th/0009052.

18. L. W. Mo and Y. S. Tsai, Rev. Mod. Phys. 41, 205 (1969).

19. M. N. Rosenbluth, Phys. Rev. 79, 615 (1950).

20. A. I. Akhiezer and M. P. Rekalo, Dokl. Akad. Nauk USSR 180, 1081 (1968); Sov. J. Part. Nucl. 4, 277 (1974).

21. L. Andivahis et al., Phys. Rev. D50, 5491 (1994).

22. M. K. Jones et al., Phys. Rev. Lett. 84, 1398 (2000); O. Gayou et al., Phys. Rev. Lett. 88, 092301 (2002).

23. P. A. M. Guichon and M. Vanderhaeghen, Phys. Rev. Lett. 91, 142303 (2003).

24. P. A. M. Guichon and M. Vanderhaeghen, Phys. Rev. Lett. 91, 142303 (2003); P. G. Blunden, W. Melnitchouk and J. A. Tjon, Phys. Rev. Lett. 91, 142304 (2003); Y.-C. Chen, A. Afanasev, S. J. Brodsky, C. E. Carlson and M. Vanderhaeghen, Phys. Rev. Lett. 93, 122301 (2004); M. P. Rekalo, E. Tomasi-Gustafsson, Nucl. Phys. A740, 271 (2004), A742, 322 (2004).

25. J. Arrington, P. G. Blunden, and W. Melnitchouk, arXiv:1105.0951.

26. J. Gunion, L. Stodolsky, Phys. Rev. Lett. 30, 345 (1973).

27. V. Franko, Phys. Rev. D8, 826 (1973).

28. V. N. Boitsov, L.A. Kondratyuk, and V. B. Kopeliovich, Sov. J. Nucl. Phys. 16, 238 (1973).

29. F. M. Lev, Sov. J. Nucl. Phys. 21, 45 (1975).

30. M. P. Rekalo, Egle Tomasi-Gustafsson, D. Prout, Phys. Rev. C60, 042202 (1999).

31. A. P. Kobushkin et al., arXiv: 1109.3562 [nucl-th].

32. I. V. Akushevich and N. M. Shumeiko, J. Phys. G: Nucl. Part. Phys. 20, 513 (1994).

33. S. Y. Choi, T. Lee and H. S. Song, Phys. Rev. D 40, 2477 (1989).

34. G. I. Gakh, N. P. Merenkov, Pis'ma v ZhETF 73, 659 (2001).

35. G. I. Gakh, N. P. Merenkov, JETP 98, 853 (2004).

36. M. Gourdin, C. A. Piketty, Nuovo Cim. 32, 1137 (1964).

37. M. Gourdin, Phys. Rep. 11C, 29 (1974).

38. M. J. Moravcsik and P. Ghosh, Phys. Rev. Lett. 32, 321 (1974).

39. I. Kobzarev, L. B. Okun' and M. V. Terent'ev, JETP Lett. 2, 289 (1965). Dubovik, E. P. Likhtman and A. A. Cheshkov, JETP 25, 464 (1967).

40. H. S. Song, F. L. Ridener, Jr. and R. H. Good, Jr., Phys. Rev. 25 D, 61 (1982). 
41. R. G. Arnold, C. E. Carlson and F. Gross, Phys. Rev. C23, 363 (1981).

42. P. J. Mohr and B. N. Taylor, Rev. Mod. Phys. 72, 351 (2000).

43. T. E. O. Ericson and M. Rosa-Clot, Nucl. Phys. A405, 497 (1983).

44. D. Schildknecht, Z. Phys. 185, 382 (1965); 201, 99 (1967); Phys. Lett. 10, 254 (1964); H. Arenhovel, S. K. Singh, Eur. Phys. J. A 10, 183 (2001).

45. A. V. Afanasev, I. Akushevich and N.P. Merenkov, JETP 125, 462 (2004).

46. Bates FPP Collaboration, B.D. Milbrath et al., Phys. Rev. Lett. 80, 452 (1998); 82, 2221(E) (1999). Jefferson Lab Hall A Collaboration, M.K. Jones et al., Phys. Rev. Lett. 84, 1398 (2000).

47. A. Akhundov, D. Bardin, L. Kalinovskaya, and T. Riemann, Fortch. Phys. 44, 373 (1996); J. Blumlein, Phys. Lett. B271, 267 (1991); J. Blumlein, Z. Phys. C65, 293 (1995).

48. A. V. Afanasev, I. Akushevich, A. Ilyichev, and N. P. Merenkov, Phys. Lett. B514, 269 (2001).

49. Y. B. Dong, C. W. Kao, S. N. Yang, Y. C. Chen, Phys. Rev. C74, 064006 (2006).

50. G. I. Gakh, E. Tomasi-Gustafsson, Nucl. Phys. A799, 127 (2008).

51. Yu Bing Dong, D. Y. Chen, Phys. Lett. B675, 426 (2009).

52. E.A. Kuraev, N.P. Merenkov, F.S. Fadin, Yad. Fiz. 47, 1593 (1988) [(Sov. J. Nuc. Phys. 47, 1009) (1988)].

53. E.A. Kuraev, F.S. Fadin, Yad. Fiz. 41, 733 (1985) [Sov. J. Nuc. Phys. 41, 466) (1985)].

54. S. Jadach, M. Skrzypek, and B. F. L. Ward, Phys. Rev. D47, 3733 (1993).

55. M.I. Konchatnij, N.P. Merenkov, O.N. Schekhovtsova, Zh. Eksp. Teor. Fiz. 118, 5 (2000)[JETP 93, $1(2000)]$.

56. G. I. Gakh and O. N. Shekhovtsova, JETP 99, 898 (2004).

57. The Jefferson Lab $t_{20}$ Collaboration, D. Abbott et al., Eur. Phys. J. A7, 421 (2000); wwwdapnia.cea.fr/SphnT20.

58. A. P. Kobushkin and A. I. Syamtomov, Phys. At. Nucl. 58, 1477 (1995).

59. A. Airapetian et al., Phys. Rev. Lett. 95, 242001 (2005).

60. The Madison Convention. Proc. of the 3-d International Symposium on Polarizatiion Phenomena in Nuclear Physics, Madison, 1970, ed. by H. H. Berschall and W. Haeberli, University of Wisconsin Press, Madison, WI, 1971, p. xxv. 